\title{
Single mosquito metatranscriptomics identifies vectors, emerging pathogens and reservoirs in one assay
}

Joshua Batson, Gytis Dudas, Eric Haas-Stapleton, Amy L. Kistler, Lucy M. Li, Phoenix Logan, Kalani Ratnasiri, Hanna Retallack

\begin{abstract}
Mosquitoes are major infectious disease-carrying vectors. Assessment of current and future risks associated with the mosquito population requires knowledge of the full repertoire of pathogens they carry, including novel viruses, as well as their blood meal sources. Unbiased metatranscriptomic sequencing of individual mosquitoes offers a straightforward, rapid and quantitative means to acquire this information. Here, we profile 148 diverse wild-caught mosquitoes collected in California and detect sequences from eukaryotes, prokaryotes, 24 known and 46 novel viral species. Importantly, sequencing individuals greatly enhanced the value of the biological information obtained. It allowed us to a) speciate host mosquito, b) compute the prevalence of each microbe and recognize a high frequency of viral co-infections, $c$ ) associate animal pathogens with specific blood meal sources, and d) apply simple co-occurrence methods to recover previously undetected components of highly prevalent segmented viruses. In the context of emerging diseases, where knowledge about vectors, pathogens, and reservoirs is lacking, the approaches described here can provide actionable information for public health surveillance and intervention decisions. (167 words)
\end{abstract}

\section{Introduction}

Mosquitoes are known to carry more than 20 different eukaryotic, prokaryotic, and viral agents that are pathogenic to humans (WHO, 2017). Infections by these mosquito-borne pathogens account for over half a million human deaths per year, millions of disability-adjusted life years (GBD 2017 Causes of Death Collaborators, 2018; GBD 2017 DALYs and HALE Collaborators, 2018; GBD 2017 Disease and Injury Incidence and Prevalence Collaborators, 2018), and periodic die-offs of economically important domesticated animals (Nonito Pagès and Lee W. Cohnstaedt, 2018). Moreover, recent studies of global patterns of urbanization and warming, as well as the possibility of mosquito transport via long-range atmospheric wind patterns point to an increasing probability of a global expansion of mosquito habitat and a potential concomitant rise in mosquito-borne diseases within the next 2-3 decades (Huestis et al., 2019; Kraemer et al., 2019). While mosquito control has played a major role in eliminating transmission of these diseases in many parts of the world, costs and resources associated with basic control measures, combined with emerging pesticide resistance, pose a growing challenge in maintaining these gains (Wilson et al., 2020).

Female mosquitoes take up blood meals from humans and diverse animals in their environment and serve as a major source of trans-species introductions of infectious microbes. For well-studied mosquito-borne human pathogens such as West Nile virus, an understanding of the transmission dynamics between animal reservoir, mosquito vector, and human hosts has been essential for public health monitoring and intervention (Hofmeister, 2011). In contrast, transmission dynamics are less clear 
for emerging microbes with pathogenic potential. Metagenomic sequencing of individual mosquitoes provides a means to comprehensively identify mosquito species, the pathogens they carry and the animal hosts that define a transmission cycle with a single assay.

We also lack a comprehensive understanding of the composition of the endogenous mosquito microbiota, which has been suggested to impact the acquisition, maintenance, and transmission of pathogenic mosquito-borne microbes. For example, Wolbachia, a highly prevalent bacterial endosymbiont of insects (Werren et al., 2008) has been shown to inhibit replication of various mosquito-borne, human-pathogenic viruses when introduced into susceptible mosquitoes (Moreira et al., 2009). These observations have led to the development of Wolbachia-based mosquito control programs for Aedes aegypti mosquitoes, which vector yellow fever virus, dengue virus, Zika virus, and chikungunya virus. Experimental releases of Aedes aegypti mosquitoes transinfected with Wolbachia have resulted in a significant reduction in the incidence of dengue virus infections in local human populations. Laboratory-based studies have identified additional endogenous mosquito microbes, such as midgut bacteria and several insect-specific flaviviruses. Greater knowledge of these endogenous microbes could inform their potential use in interfering with mosquito acquisition of and competence to transmit pathogenic Plasmodium species and human flaviviruses, respectively. Quantitative analysis of the composition of endogenous microbes and the viruses in individual mosquitoes would be needed to establish a role for these agents in naturally occurring infections and/or transmission of known human pathogens.

Several recent, unbiased metagenomic analyses of batches of mosquito pools collected around the world have begun to address these issues (Atoni et al., 2018; Fauver et al., 2016; Frey et al., 2016; Li et al., 2015; Moreira et al., 2009; Pettersson et al., 2019; Sadeghi et al., 2018; Shi et al., 2019, 2015, 2017, 2016; Xia et al., 2018; Xiao et al., 2018a, 2018b) (reviewed in (Atoni et al., 2019; Xiao et al., 2018b)). These studies, which have primarily focused on analysis of viruses, have expanded our understanding of the breadth of viral diversity present in mosquito populations worldwide. However, they have not provided key epidemiologic information needed to direct intervention. This includes the measurement of viral prevalence within mosquito populations, their potential reservoir sources, or the impact that additional bacterial and eukaryotic microbes carried by mosquitoes might have on virus carriage, transmission, and pathogenesis.

Single mosquito analyses are required to link blood meal sources, endogenous microbes, and co-occurring pathogens. A handful of small-scale studies have demonstrated that it is possible to identify divergent viruses and evidence of other microbes in single mosquitoes via metagenomic next-generation sequencing (Bigot et al., 2018; Chandler et al., 2015; Shi et al., 2019). Here, we analyzed the metatranscriptomes of 148 individual mosquitoes collected in California, USA. We characterized the composition of their co-infecting microbes, quantified the prevalence and load of detectable viruses and selected bacterial and eukaryotic microbes, and identified blood meal sources and their associated pathogens. Crucially, sequencing a large number of individuals allowed for simple co-occurrence analyses that extended the sensitivity to detect missing or as-yet unidentified viral genome segments with no recognizable homology to previously described sequences. Our findings demonstrate how large-scale single mosquito metatranscriptomics can define both the mosquito's complex microbiota, including mosquito-borne pathogens, and its blood meal sources, thus contributing critical epidemiological information needed to control transmission. 


\section{Results}

\section{Mosquito host speciation by comparative whole transcriptome analysis}

Adult Aedes, Culex, and Culiseta mosquito species circulating in California in late fall of 2017 were collected to acquire a diverse and representative set of 148 mosquitoes for metatranscriptomic next generation sequencing (mNGS) analysis. We targeted collections across a variety of habitats within 5 geographically distinct counties in Northern and Southern California (Supplemental Figure 1). Visual mosquito species identification was performed at the time of collection (Materials and Methods, results are summarized in Table S1). Primarily female mosquitoes were included to enrich for blood-feeding members of the population responsible for transmission of animal and human diseases. Total RNA extracted from each mosquito was used as the input template for mNGS to capture both polyadenylated and non-polyadenylated host, viral, prokaryotic, and eukaryotic RNAs (Materials and Methods; per mosquito sequence yields are summarized in Table S2).

Given the important role of accurate identification of mosquito species for understanding geospatial mosquito circulation and vector-pathogen interactions, and the potential for human error in visual inspection, we investigated if single mosquito mNGS could provide a complementary, unbiased molecular method for identifying mosquito species. Because complete genome sequences were not available for all mosquito species identified visually in this set, we applied a reference-free, kmer-based approach (Harris, 2018) to compute pairwise genetic distances between the complete metatranscriptomes acquired for each of the 148 mosquitoes. Samples were grouped using hierarchical clustering and the most common visually identified species within each group was taken as a consensus species call for that group (Figure 1 and see Supplemental Figure 2 for detailed alignment of visual calls with the clustered genetic distance matrix, and Supplemental Data File 1 for underlying snp distance data). These molecular groupings of mosquito genera and species agreed the visual calls for $95 \%$ of the specimens ( $n=140 / 147$, one sample had no visual identification). The discordant calls occurred in 2 contexts reported to present challenges to morphology-based speciation: 1) within the Culex genus in which genetic hybridization among species members has been documented and reported to confound accurate morphological speciation in California (Cornel et al., 2003, 2003; Kothera et al., 2012; McAbee et al., 2008); and 2) between samples belonging to the Culex and Culiseta genera that share some overlap in morphology, and require detection of features (perspiracular bristles and subcostal wing vein bristles) that can be lost or damaged during trapping and handling (Darsie and Ward, 2016). Thus, we used the transcriptome-based species calls for this study. There is additional within-species structure visible on the hierarchical clustering tree that, for Aedes aegypti and Culex erythrothorax, coincides with geographic structure, raising the possibility that molecular methods may also provide insight into the distribution of subspecies as well (Figure 1). Taken together, these data show that comparative transcriptome analysis of single mosquito $\mathrm{mNGS}$ data can provide critical information regarding the identity and diversity of circulating mosquitoes. 


\section{Comprehensive and quantitative analysis of non-host sequences detected in single mosquitoes}

To understand the distribution of microbial species within the microbial cargo of mosquitoes, we first examined the overall proportion of non-host reads assembled into contigs that could be assigned to viral, bacterial, and eukaryotic taxa. A detailed overview of the analysis we applied to identify, assemble, classify, and quantify all the non-host contigs and their associated read counts is provided in Materials and Methods (summarized in Supplemental Figure 3). Details of the per mosquito breakdown of non-host read assignment across high level taxonomic categories are provided in Supplemental Figure 4 (underlying data for Supplemental Figure 4 can be found in Supplemental Data file 3). Figure 2 provides a quantitative treemap overview of how the assembled non-host reads mapped across the viral, prokaryotic, and eukaryotic taxa (see Supplemental Data File 2 for data underlying Figure 2 and Supplemental Figure 5, a higher resolution treemap overview). In sum, we were able to classify, to at least kingdom level, $77 \%$ of the 13 million non-host reads that assembled into contigs with more than two reads.

\section{Diverse known and novel RNA virus taxa dominate the mosquito microbiota.}

We found that the vast majority of the non-host reads of mosquitoes assembled into contigs corresponding to complete viral genomes (11 million reads of 13 million total non-reads; Figure 2, all blocks in the treemap annotated with suffix "-viridae"). Positive sense single stranded RNA viruses made up the most abundant class of detected viruses ( 7.4 million reads of the 11 million viral reads; Figure 2 blocks labeled Solemoviridae, Luteoviridae, Tombusviridae, Narnaviridae, Flaviviridae, Virgaviridae, and Filovirida), negative sense single stranded RNA viruses made up the next most abundant virus category (2.25 million reads of the 11 million viral reads; Figure 2 blocks labeled Peribunayviridae, Phasmaviridae, Phenuiviridae, Orthomyxoviridae, Chuviridae, Rhabdoviridae, and Ximnoviridae), and double-stranded RNA viruses formed the third most abundant virus category $(0.94$ million reads of the 11 million reads; Figure 2 blocks labeled Chrysoviridae, Totiviridae, Partitiviridae, and Reoviridae). In many cases, multiple independent isolates of complete viral genomes were recovered across the individual mosquito specimens. In all, a total of 70 distinct viral taxa were recovered, 46 of which correspond to distinctly divergent novel viruses (Table 1). Intriguingly, only 10 of the 24 previously described viral taxa have been previously recovered from mosquitoes in California (Table 1, rows highlighted in gray (Chandler et al., 2015; Sadeghi et al., 2018)). Although a number of the known and novel viral species we detected corresponded to additional viral species from families previously thought to only infect plants and fungi, (e.g., the Chrysoviridae, Totiviridae, Luteoviridae and Solemoviridae, Table 1), emerging evidence from mosquito and broader insect metatranscriptomics has indicated that these viral taxa are tightly associated with, if not actually infecting, mosquitoes and other insects (Shi et al., 2017, 2016; Xiao et al., 2018b).

The balance of additional reads that could be assigned to viral taxa corresponded to reads assembled into contigs that were clearly viral in origin but incomplete by either not associated with an RNA-dependent polymerase ( 0.37 million reads, Figure 2, light gray block labeled "uncurated viruses") or associated with contigs aligning to viral taxa that were detected at levels too low to visualize on the treemap. This latter set of low abundance viral taxa corresponded to several types of DNA viruses, such as nucleocytoplasmic large DNA viruses, members of the Polydnaviridae, Alphabaculovirus, Nudiviridae, and Circovirus-like sequences, and phages (data not shown). Some of these low abundance viral taxa reflect bona fide infections, while others are likely the result of indirect infections. For example, 6 
distinct types of Botourmiaviridae, a family of viruses primarily known to infect fungi, were all detected at very low levels in a single mosquito that was also found to be co-infected with a more likely Botourmiaviridae host species, the ergot fungus, Claviceps.

\section{Trypanosomatidae and vertebrate species are major constituents of the eukaryotic taxa.}

An additional 2.2 million of the 13 million non-host reads assembled into contigs that mapped to non-viral taxa. Just under 1 million reads could be assigned to eukaryotic taxa ( 0.9 million reads total, Figure 2, bottom left row of boxes). Members of Trypanosomatidae comprised $50 \%$ of these reads. Slightly more than half ( 0.25 million reads) of the Trypanosomatidae reads correspond to the subfamily Leishmaniinae, that encompasses multiple species known to infect insects and vertebrates. The second most abundant group of eukaryotes detected in the dataset were Bilateria (animals), with 0.20 million reads made up of mammals (Boreoeutheria, 73,000 reads) and birds (Aves, 51,000 reads), followed by invertebrates (Ecdysozoa, 36,000 reads (not shown); see Supplemental Figure 5 for higher resolution details of these and other notable lower abundance eukaryotic taxa detected). The reads derived from vertebrate taxa almost certainly belong to blood meal hosts, which we investigate in detail below. Fungal and plant contigs, made up the remainder of the eukaryotic reads we captured from individual mosquito sequencing, with 79,000 and 62,000 total reads, respectively.

\section{Wolbachia species make up the majority of prokaryotic taxa.}

Prokaryotic contigs encompassed 0.7 million non-host reads. Among the prokaryotic taxa detected, Wolbachia, a known endosymbiont of Culex quinquefasciatus (Werren et al., 2008), comprised most of the reads ( 0.22 million reads, Figure 2, bottom central row of brown-hued blocks). Various other bacterial taxa were detected at lower abundance; i.e. members of Alphaproteobacteria, Gammaproteobacteria, Terrabacteria group, and Spirochaetes (Spironema culicis (73,000 reads), a bacterial species previously detected in Culex mosquitoes (Cechová et al., 2004; Duguma et al., 2019), makes up $68 \%$ of Spirochaete reads). A higher resolution overview of the lowest common ancestor (LCA) species we could assign within each of these 4 broad categories is provided in Supplemental Figure 5 and Supplemental Data File 2. Interestingly, these results largely agreed with data obtained for the Culex and Aedes species in prior sequencing studies involving more directed capture of prokaryotic and eukaryotic taxa via 16S rRNA metabarcoding of mosquitoes collected in Thailand (Thongsripong et al., 2018).

\section{Ambiguous and metagenomic "dark matter" sequences are present.}

A significant portion of the non-host reads assembled into contigs with sequences that were taxonomically ambiguous. Approximately 0.5 million reads assembled into contigs with a lowest common ancestor (LCA) assigned to the taxonomy nodes of "root" or "cellular organisms" (Figure. 2, unlabeled light gray box). A much larger fraction of non-host reads -- approximately 4.4 million reads -corresponded to metagenomic "dark matter", i.e. contigs without any recognizable sequence homology to previously published sequences. Contig co-occurrence analysis across the individual mosquito sequence results (see main text below) allowed us to identify additional viral contigs from this set of 
contigs, contributing 0.4 million reads to the total tally of detectable viral reads in the mosquito microbiota.

Together these data establish the utility of our comprehensive single mosquito mNGS analyses to define the composition and diversity of the mosquito metatranscriptome. The sensitivity of our analysis reveals endogenous constituents of the mosquito microbiome, the source of their blood meals, and the potential human and animal pathogens they carry, and even viruses that selectively infect mosquito-associated fungi.

\section{Identifying constituents of the mosquito microbiota}

To define components of the mosquito microbiota and investigate the potential variation between individuals, we next analyzed the distribution of the viral, prokaryotic, and microbial eukaryotic taxa detectable across individual mosquitoes. All confidently called contigs for microbial taxa detected within each mosquito were compiled, and the fraction of non-host reads aligning to each contig was computed to estimate the composition and proportions of microbial agents detectable within each mosquito. Figure 3 displays those agents that were detectable at a level above $1 \%$ of non-host reads plotted as bars for each individual mosquito analyzed. The data for read counts per taxon per sample and detected viral taxa can be found in Supplemental Data Files 4 and 5, respectively. Notably, given the sensitivity of our data and analytical pipeline (see Materials and Methods), we could confidently call contigs even when supported by $<1 \%$ of the non-host reads (i.e., $<<0.02 \%$ of total reads). These are plotted as dark circle symbols above the x-axis coordinate for the central Wolbachia panel of Figure 3 , and grey bars in the Trypanosomatidae, Apicomplexa, and Nemotoda panels at the base of Figure 3 (underlying data for these selected bacterial and eukaryotic microbial taxa are provided in Supplemental Data Files 6 and 7, respectively). The data reveal unprecedented insight into the heterogeneity in the microbiota associated with individuals, between mosquito species and collection sites.

\section{Viral diversity and prevalence measured in single mosquitoes}

Given their predominance and potential relevance to human disease, we first examined the 70 unique viral taxa on a single mosquito basis (Table 1, Supplemental Data File 4). Of these, only 24 were closely related or identical to previously identified mosquito viruses. The remaining 46 viral genomes shared less than $85 \%$ amino acid sequence identity to any publicly available viral sequences. Despite this divergence, family-level sequence conservation of genomic features allowed us to confirm complete genome sequence recovery. For example, conserved sequences were identified at the $5^{\prime}$ and $3^{\prime}$ ends of bunyavirus segments in a novel peribunya-like virus (Udune virus, Table 1 ) with $28 \%$ to $76 \%$ amino acid identity to its closest relative (Supplemental Figure 6 and Supplemental Data File 8). Only a single mosquito harbored this virus, and at a relatively low abundance of reads ( $0.02 \%$ of total reads). Combining this approach with the co-occurrence investigation detailed below (Figure 6 and main text), we were able to identify and assign the 45 additional novel genomes to specific viral taxa. Thus, single mosquito analysis provides a highly sensitive approach to detect new and divergent viral species circulating in mosquito populations, even when present at low prevalence or abundance.

Single mosquito analysis also shed light on the variability in the composition and number of viral reads both within and across mosquito species (Figure 3, top panel), and their corresponding collection sites (indicated by colored bar plot at the bottom of the plots). Importantly, among single mosquitoes, 
co-infections predominated, with $88 \%$ of mosquitoes harboring 2 or more (median 3 ) distinct viral taxa (Figure 4A, see Supplemental Data File 9 for underlying data). Focused analysis of viral species within single mosquitoes provides the opportunity to examine the proportion of viruses within each of these co-infections, which in turn can inform and extend our understanding of the distribution patterns of known and emerging novel viruses within the mosquito population, and the associated co-infecting viruses. Figure 3 , shows the wide range in the number and type of viruses that are detected across individual mosquitoes. For instance, several Culex mosquito species stand out as outliers harboring only a single viral species encompassing $>50 \%$ of the non-host reads assembled into contigs in that mosquito (Figure 3, top panel: Iflaviridae species [dark green bars] in Culex tarsalis; Tombusviridae and Virgaviridae species [light blue bars] in Culex erythrothorax). At the other end of the spectrum are multiple examples of individual mosquitoes that do not stand out with regard to viral read abundance, yet still harbor a mixture of 4 or more viruses (Figure 3, top panel - see especially Culex tarsalis and Culiseta species plots). Other viruses that are detected broadly across diverse mosquito species (Figure 3, top panel, see the Solemoviridae/Luteoviridae [yellow bars], Narnaviridae [blue bars], Virgaviridae [light blue bars], and Dicistroviridae/Iflaviridae [dark green bars]). Interestingly in some mosquito species, these viruses are the predominant proportion of the non-host reads assembled into viral contigs, while in other mosquito species where these viruses are detected, they make up only a minor fraction. The opposite pattern of virus distribution where viral species are restricted to a single mosquito species, for example the Partitiviridae and Reoviridae among the Culiseta species (Figure 3, top panel, right edge of plot, dark purple bars). These distinct patterns of viral distribution point to potentially testable hypotheses as to their causes, such as mosquito species susceptibility or competence to vector a virus, the potential pathogenicity of a given virus (or mixture of viruses), or factors in the environment, such as food sources or weather. Regardless of the ultimate source of this variability, such insights are only possible by analyzing mosquitoes individually rather than in bulk.

This variation is particularly relevant when we consider that viral abundance is often calculated based on bulk mosquito sequencing, which does not provide information about the prevalence or heterogeneity in abundance of a virus across the mosquito population. Importantly, we find that the average abundance of a virus (i.e. the average number of reads across a set of mosquitoes) is not necessarily predictive of the prevalence of that virus (i.e. the number of mosquitoes in which it occurs). For example, Culex narnavirus 1 and Culex pipiens-associated Tunisia viruses were found at similar abundance in Culex erythrothorax mosquitoes obtained from the same collection site in West Valley; however, the latter was 3 times more prevalent (30\% vs 90\%, Figure 4B; see Supplemental Data File 10 for underlying data). A more global view of viral diversity and prevalence across mosquito species is shown in (Figure 4C and summarized in Supplemental Data File 11) which plots the fraction of individuals infected for each mosquito species and each virus detected in our study. This quantitative and comprehensive analysis of the prevalence of mosquito-borne viruses would not be possible without single mosquito sequencing, yet provides critical epidemiological information needed to manage the transmission of mosquito-borne viruses. For example, the sampled mosquito genera (Culex, Aedes, and Culiseta) have distinct viromes, with only four viruses shared across genus boundaries and even then, only Merida virus (-ssRNA) and Culex iflavi-like virus 4 (+ssRNA) are shared by Aedes and Culex mosquitoes. Within each genus, viruses appear to be largely unique to species, though some overlap is detectable (Figure 4C), potentially reflecting greater similarities in ecology and physiology (Longdon et al., 2014) that enable an easier flow of viruses between populations. 


\section{Exploring the impact of Wolbachia endosymbionts}

We restricted our single mosquito analysis of detectable prokaryotes to Wolbachia given its abundance and evidence suggesting that as an endosymbiont it could impact the microbiota of its mosquito hosts. Wolbachi was detected in 32 mosquitoes belonging to Culex quinquefasciatus, Culex pipiens, and Aedes albopictus species (Figure 3, middle panel, black bars and circle symbols; see Supplemental Data File 6 for underlying data). These observations are consistent with previous reports of wild-caught mosquito species that are naturally infected with Wolbachia (Kittayapong et al., 2000; Rasgon and Scott, 2004). Among these 3 species, Wolbachia was detected in all or nearly all of the mosquitoes. Thus, it was not possible to draw definitive conclusions regarding whether the presence or absence of Wolbachia influenced the composition of detectable co-occurring viral taxa among these mosquitoes. However, the fraction of non-host reads assigned to Wolbachia per mosquito varied dramatically, from $<1 \%$ to $74 \%$ (Figure 3, middle panel, black circles and black bar plots, respectively), and revealed interesting trends that would require further validation. For example, for Ae. albopictus, individuals with higher levels of detectable Wolbachia (Figure 3, central panel: samples with black bars) exhibited lower numbers of reads from single stranded positive sense RNA viruses (Solemonviridae and Luteoviridae) than individuals with a lower percentage of Wolbachia reads (Figure 3, central panel: samples with black circles). Similarly, higher levels of Wolbachia in Culex pipiens mosquitoes also generally correlated with lower viral abundance. Although not statistically significant, given the low sample numbers and lack of Wolbachia positive and negative individuals, these data again demonstrate the potential utility of sequencing individuals.

\section{Prevalence of eukaryotic microbes and pathogens in single mosquitoes}

Although we detected fungi, plants and other eukaryotes in our analyses (Figure 2), we focus here on three potentially human pathogenic species: Trypanosomatidae, which was the most abundant eukaryotic taxon detected and contains established pathogens of both humans and birds; Apicomplexa, which encompasses the causative agents of human and avian malaria; and Nematoda, which contain filarial species that cause heartworm in canines and filarial diseases in humans (Supplemental Data File 7).

Twelve mosquitoes (8\%) were found to harbor Trypanosomatidae taxa (Figure 3, bottom panel). We detected sequences corresponding to monoxenous (e.g. Crithidia and species), dixenous (Trypanosoma, Leishmania species), as well as the more recently described Paratrypanosoma confusum species. Of the Trypanosomatidae-positive mosquitoes, eight were Culex erythrothorax mosquitoes, while the remaining four were Culex pipiens and 2 Culex tarsalis Figure 3, bottom panel). Notably, all were collected from the same trap site in Alameda County, albeit at different times, providing insight into, in this case, a limited distribution and potential prevalence of Trypanosomatidae within the mosquito population.

We investigated the distribution of the Apicomplexa contigs and reads, as this phylum encompasses the Plasmodium genus, which includes several pathogenic species that cause avian and human malaria. Within our single mosquito dataset, we identified 8 mosquitoes with Apicomplexa contigs (Figure 3, bottom panel). These corresponded to 3 Aedes aegypti mosquitoes and 1 Culex quinquefasciatus mosquito, both collected in San Diego, and 2 Culex erythrothorax mosquitoes, 1 Culex pipiens mosquito, and 1 Culex tarsalis mosquito collected in Alameda County. Only the Culex quinquefasciatus mosquito 
harbored Apicomplexa reads at a level above $1 \%$ of total non-host reads. Interestingly, this mosquito harbored Wolbachia, but no viruses could be detected.

Finally, we examined taxa falling under Nematoda, a phylum that encompasses a diverse set of more than 50 filarial parasites of humans and animals. Here, we saw evidence of Nematoda carriage in 3 Culex mosquitoes: 2 Culex tarsalis and 1 Culex pipiens (Figure 3, bottom panel). Two of these mosquitoes were collected in Alameda County and showed very low levels Nematoda ( $<1 \%$ of non-host reads, Figure 3 , bottom panel, dark gray square symbols). In the third mosquito, a Culex tarsalis collected in Coachella Valley, the Nematoda made up $2 \%$ of the non-host reads (Figure 3, bottom panel gray bar with red outline).

Together these data reveal the diversity and prevalence of microbial species harbored within single mosquitoes and establish the comprehensive nature and sensitivity of single mosquito metagenomic analysis.

\section{Blood meals and associated microbes}

As vectors, mosquitos transfer the pathogenic microbes they carry from one animal to another as they feed. Identifying the sources of these blood meals can provide critical information regarding the animal reservoir of these vector-pathogens and the paths of transmission. Therefore, we next investigated the possibility of identifying the blood meal host directly from mNGS. We restricted this analysis to the 60 mosquitoes from Alameda County, as they were selected for visible blood-engorgement. For 45 of the 60 mosquitoes, there was at least one contig with an LCA assignment to the phylum Vertebrata (range $=$ 1-11 contigs, with 4-12,171 supporting reads). To assign a blood meal host for each of these mosquitoes, we compiled their corresponding Vertebrata contigs and selected the lowest taxonomic group consistent with those contigs. For all samples, the blood meal call fell into one of five broad categories (Figure 5, underlying data and detailed summary provided in Supplemental Files 12 and 14, respectively): even-toed ungulates (Pecora), birds (Aves), carnivores (Carnivora), rodents (Rodentia), and rabbits (Leporidae). For 10 samples, we were able to identify the genomic source at the species level, including rabbit (Oryctolagus cuniculus), mallard duck (Anas platyrhynchos), and raccoon (Procyon lotor).

The potential blood meal sources identified were broadly consistent with the habitats where the mosquitoes were collected. For the 25 samples collected in or near the marshlands of Coyote Hills Regional Park, we compare our calls to the wildlife observations in iNaturalist, a citizen science project for mapping and sharing observations of biodiversity. iNaturalist reports observations consistent with all five categories, including various species of squirrel, rabbit, raccoon, muskrat, and mule deer. The mosquitoes with blood meals in Pecora are likely feeding on mule deer, as no other ungulate commonly resides in that marsh (iNaturalist, 2020).

We also investigated whether bloodborne pathogens of the blood meal source were detectable. We performed a hypergeometric test for association between each blood meal category and each microbial taxon (see Materials and Methods and Supplemental Data File 13). The only statistically significant association ( $p=0.0005$, Bonferroni corrected) was between Pecora and Anaplasma, an intracellular erythroparasite transmitted by ticks. Anaplasma was detected in 11 of the 20 samples with Pecora. This striking co-occurrence suggests a possible burden of anaplasmosis in the local deer population.

Additionally, we detected evidence for three other bloodborne pathogens which, because of the small number of observations, could not pass the threshold of statistical significance. These included an orbivirus closely related to those known to infect deer, a Trypanosoma species previously found in birds, 
and the apicomplexans Plasmodium and Eimeria from species known to infect birds (See Materials and Methods). The likely hosts of these pathogens were also concordant with the blood meal calls. Thus, sensitive and comprehensive metagenomic analysis of single mosquitoes not only provides information as to paths of transmission, it also provides a tool to detect emerging pathogens within animal communities in their environments.

\section{Recovery and assignment of previously unrecognizable viral genome segments and species within the orthomyxovirus family}

Although many new viruses can be identified in bulk samples, the majority of these are identified only via their conserved RNA-dependent RNA polymerase (RdRp). Recovering complete genomes for segmented viruses from bulk samples is challenging, as genes that are not highly conserved may be unrecognizable by sequence homology. Moreover, the assignment of putative segments to a single genome can be confounded if the pooled libraries are derived from mosquitoes with multiple infections of related segmented viruses.

By sequencing many individual mosquitoes, we can exploit the fact that all segments of a segmented virus will co-occur in the samples where that virus is present and be absent in samples where the virus is absent. Applying these criteria to our data analysis should enable the identification of previously unidentified viral genome segments. To do this, we first grouped all contigs that were longer than 500 nucleotides into clusters of highly homologous contigs, then grouped these clusters by co-occurrence across all of the 148 individual mosquitos sampled (Figure 6A). Importantly, this required only the sequence information from the study, without using any external reference. We then scanned each cluster for sequences containing a viral RdRp domain (see Materials and Methods). For each RdRp cluster, we consider any other contig cluster whose sample group overlaps the set of samples in the viral RdRp cluster above a threshold of $80 \%$ as a putative segment of the corresponding virus. A cluster-by-sample heatmap for all segments co-occurring with RdRps resulted in 27 candidate complete genomes for segmented viruses (Supplemental Figure 7, underlying data provided in Supplemental Data Files $15 \mathrm{~A}$ and 15B). For the 79 of the 96 putative segments recognizable by homology to published sequences (colored in black), these groupings into genomes were accurate. This supports the notion that the remaining 17 putative segments (colored in red), which lack homology to any known sequences at either nucleotide or amino acid level, may indeed be part of viral genomes. Combined, these putative segments represented $8 \%$ of the metagenomic "dark matter" portion of the reads in the study.

Our co-occurrence analysis enabled the discovery of new viral segments and new viral species within the segmented Orthomyxoviridae family (Figure 6B; see Supplemental File S16 for underlying data). Orthomyxoviruses are segmented viruses (ranging from 6 to 8 segments) including influenza viruses, isaviruses, thogotoviruses, and quaranjaviruses that infect a range of vertebrate and arthropod species. Quaranjaviruses are largely found in arthropods, and in this study, we identified four quaranjaviruses, two of which were previously observed in mosquitoes collected outside California (Wuhan Mosquito Virus 6 (WMV6) (Li et al., 2015; Shi et al., 2017) and Guadeloupe mosquito quaranja-like virus 1 (GMQV1) (Shi et al., 2019)) and two, which we have named Ūsinis virus and Astopletus virus, were previously unknown.

Thus, for WMV6 and GMQV1 detected here, we observed all of the previously identified segments (Li et al., 2015; Shi et al., 2017), as well as two additional segments (which we named hypothetical 2 and hypothetical 3) for WMV6 and five for GMQV1 (PA, gp64, hypothetical, hypothetical 2, hypothetical 3) 
(Supplemental Figure 8A and 8B). We confirmed the existence of the 2 putative segments for WMV6 by assembling homologous segments from reads in two previously published datasets describing this virus. For GMQV1, we were able to find reads in NCBI's short read archive entries that are similar at the amino acid level to putative protein products of the two new segments; however, there was not sufficient coverage to reconstruct whole segments. Furthermore, phylogenetic trees constructed separately for each of the eight segments of WMV6 have similar topologies (See tanglegram, Figure 7; see Supplemental Data File 17 for underlying data), suggesting that the two new putative segments have evolved in conjunction with the previous six, bringing the total number of segments for each genome to eight.

For the two quaranjaviruses discovered in this study, Ūsinis virus and Astopletus virus, the co-occurrence analysis also produced 8 segments, 5 and 4 of which, respectively, were recognizable by alignment to NCBI reference sequences. The hypothetical 2 and hypothetical 3 segments we identified from this set of four quaranjavirus genomes are too diverged from one another to align via BLASTx, but they do share cardinal features such as sequence length, ORF length, and predicted transmembrane domains (Supplemental Figure 8A-D). Intriguingly, this set of four viruses are part of a larger clade of quaranjaviruses (Supplemental Figure 9, Supplemental Data File 18). It is nearly certain that the remaining seven viruses in this clade also have eight segments and quite likely that all quaranjaviruses share this genome organization hinted at in earlier studies (Zeller et al., 1989).

The high rate of viral co-infections detected among the single mosquitoes we analyzed (Figure 4A) indicated a concomitant high likelihood that multiple mosquitoes could harbor more than one segmented virus, and potentially confound our co-occurrence analysis. However, the co-occurrence threshold of 0.8 that we applied was sufficient to deconvolve those segments into distinct genomes in

all cases but one. There were 15 mosquito samples containing both Ūsinis virus, an orthomyxovirus with eight segments (three of which were unrecognizable by BLASTx) and Barstukas virus, a Phasma-like bunyavirus, with one additional sample where only Barstukas virus was found (Figure 6B, top two blocks). In this case, we were able to disentangle the genomes of these two viruses using additional genetic information: Barstukas virus contains all three segments expected for a bunyavirus (L, GP, and NP), all of which had BLASTx hits to other Phasma-like viruses, while the unrecognizable segments of Usinis virus shared features with the other quaranjaviruses in the study (as described above).

\section{Co-occurrence reveals unknown genome segments of Culex narnavirus 1}

Beyond detection of missing genome segments for known segmented viruses, the co-occurrence analysis also revealed additional genome segments in "dark matter" contig clusters for viruses with genomes previously considered to be non-segmented. A striking example is an 850 nucleotide contig cluster that co-occurred with the Culex narnavirus 1 RdRp segment in more than 40 mosquitoes collected from diverse locations across California (Supplemental Figure 7, Supplemental Data Files 15A and 15B). Like the RdRp segment, the putative new second segment shares the exceptional feature of ambigrammatic open reading frames (ORFs), i.e. a distinct ORF encoded by the reverse complementary RNA strand (Supplemental Figure 8E). The phylogenetic tree topology for the set of 42 putative second segments is similar to the tree for the RdRp segments, suggesting co-inheritance (Supplemental Figure 10A, Supplemental Data File 19). Moreover, we were able to recover nearly identical contigs from previously published mosquito datasets, all of which also contained the Culex narnavirus $1 \mathrm{RdRp}$ segment. This provides strong evidence that this otherwise unrecognizable sequence is a genuine Culex 
narnavirus 1 segment, which we refer to here as the "Robin" segment, given it's consistent, but underappreciated presence.

Since the Narnaviruses were first described in fungi (Hillman and Cai, 2013) and recent studies have shown other eukaryotes can serve as Narnavirus hosts (Charon et al., 2019; Dinan et al., 2019; Göertz et al., 2019; Richaud et al., 2019), we investigated whether this virus co-occurred with a potential non-mosquito host. However, there was no significant co-occurrence with a non-mosquito eukaryotic taxon, or between the abundance of Culex narnavirus 1 and abundance of fungi (Supplemental Figure 10B, Supplemental Data File 20). Thus, it is likely that mosquitoes serve as direct hosts of the Culex narnavirus 1, whose genomes we show here consist of two, still enigmatic, ambigrammatic RNA segments.

\section{Discussion}

We demonstrate how mNGS of single mosquitoes, together with reference-free analyses and public databases, provides-in a single assay-critical and actionable epidemiological information. This includes quantitative information regarding circulating mosquito species, pathogen prevalence, and co-occurrence of diverse known and novel viruses, as well as prokaryotes, eukaryotes, blood meal sources and their potential pathogens. We are able to identify and confirm, using public data, the existence of previously unknown segments of both known and novel viruses, focusing on four quaranjaviruses and Culex narnavirus 1 as examples. In the context of an emerging disease, where knowledge about vectors, pathogens, and reservoirs is lacking, the techniques described here can be applied to rapidly provide actionable information for public health surveillance and intervention decisions. While unbiased sequencing of individual mosquitoes is not currently practical or appropriate in all contexts, advances in lab automation and rapidly decreasing costs of mNGS technologies are expected to increase the affordability and practicality of single mosquito sequencing in the near future.

\section{Inferring biology from sequence in the context of an incomplete reference}

The power of metatranscriptomic NGS depends on the ability to extract biological information from nucleic acid sequences. For both bulk and single mosquito sequencing studies, the primary link between sequence and biology is provided by public reference databases, and thus the sensitivity of these approaches will depend crucially on the quality and comprehensiveness of those references. In practice, even the largest reference databases, such as $\mathrm{nt} / \mathrm{nr}$ from NCBI, represent a small portion of the tree of life. Consequently, sequences derived from a sample of environmental or ecological origin, often exhibit only a low percent identity to even the best match in a database. Here, we manage that uncertainty by assigning a sequence to the lowest common ancestor of its best matches in the reference database. However, there is a fundamental limit to the precision of taxonomic identification from an incomplete reference.

An advantage of single mosquito sequencing is that it offers an orthogonal source of information: the ability to recognize nucleic acid sequences detected in many samples even when they have no homology to a reference sequence. This allowed us to associate unrecognizable sequences with viral polymerases, generating hypothetical complete genomes. The strategy of linking contigs that co-occur across samples is utilized in analysis of human and environmental microbiomes, where it is referred to as "metagenomic binning" (Breitwieser et al., 2019; Roumpeka et al., 2017). Using this approach, we identified previously 
unknown genome segments establishing that the genome of a large clade of quaranjaviruses (those descended from the common ancestor of WMV6 and GMQV1), like distantly related influenza A and B viruses, consists of 8 segments. We also discovered a second ambigrammatic RNA encoded by the Culex narnavirus that in retrospect was identifiable in multiple previously published mosquito datasets. In sum, we pulled $8 \%$ of the reads in the metagenomic "dark matter" fraction of our dataset into the light. The putative complete genomes we identified were supported retrospectively by public datasets and can be further validated by biological experiments or approaches such as short RNA sequencing that indicate a host antiviral response (Aguiar et al., 2015; Waldron et al., 2018).

Another advantage of single mosquito sequencing is the ability to supplement, or potentially circumvent, visual species identifications using molecular data. Accurate mosquito species identification is essential for the control of mosquito-borne diseases, as pathogen competence is often limited to a range of species, such as various Aedes species for Zika, dengue, and chikungunya viruses, and Anopheline species for malaria. Also, the primary mosquito species responsible for vectoring a disease can vary geographically--West Nile virus has been detected in 65 mosquito species, but a narrow range of Culex species drives transmission of the virus. Field validation of which mosquito species carry which pathogens in a specific geographic area informs targeted analysis and control of that species (Petersen et al., 2013). Here, though only 3 of the 10 collected mosquito species had complete genome references, it was possible to estimate pairwise SNP distances between samples in a reference-free way and perform an unsupervised clustering. The clusters were $95 \%$ concordant with the visual mosquito species calls, and discordant outliers were easy to detect and correct. This approach generalizes to any collection of metatranscriptomes containing multiple representatives of each species. Accurate mosquito identification is essential for selecting the appropriate strategy and materials to control viremic mosquitoes. In a bulk pool of mosquitoes, the microbiota from any miscalled specimens would be blended in with the correctly labeled ones, making it difficult or impossible to deconvolute host species after the fact. By correctly identifying the host range of a known or novel pathogen in a given area, vector control can be appropriately targeted for the prevention of disease.

\section{Distribution of microbes within mosquito populations}

Once sequences have been mapped to taxa, it is relatively straightforward to characterize the composition of the microbes within a circulating population of mosquitoes. This information can inform basic research and epidemiologic questions relevant for modeling the dynamics of infectious agents and the efficacy of interventions. A key parameter is the prevalence of a microbe, which cannot be inferred from bulk data. For the 70 viruses in this study, the prevalence ranged from detection in one mosquito (peribunya-like Udune virus) to detection in all 36 Culex tarsalis samples in the study (Marma virus).

For some questions, the prevalence data supplied by single mosquito sequencing is helpful for experimental design. For example, in our dataset, Wolbachia was either absent or endemic in each mosquito species sampled. Thus, although a trend between the amount of Wolbachia relative to viral diversity and load was detectable across samples that harbored Wolbachia, it was not possible to detect a statistically significant effect of Wolbachia on virome composition or abundance within any species. Nonetheless, our data establish that single mosquito sequencing could address such questions via more extensive, targeted sampling of mosquito populations where Wolbachia (or any other agent of interest) is expected to have an intermediate prevalence. This information would be invaluable, as the introduction of Wolbachia might be a useful biological agent to suppress viral transmission by mosquitoes (Moreira et al., 2009). 


\section{Blood meal sources and xenosurveillance}

The identification of blood meal hosts is important for understanding mosquito ecology and controlling mosquito-borne diseases. Early field observations were supplemented by serology, and, more recently, molecular methods based on host DNA. Currently, the most common method of blood meal identification is targeted PCR enrichment of a highly-conserved "barcode" gene, such as mitochondrial cytochrome oxidase I, followed by sequencing (RATNASINGHAM and HEBERT, 2007; Reeves et al., 2018). To monitor specific relationships between mosquito, blood meal, and pathogen, studies have combined visual identification of mosquitoes, DNA barcode identification of blood meal, and targeted PCR or serology for pathogen identification (Batovska et al., 2018; Boothe et al., 2015; Tedrow et al., 2019; Tomazatos et al., 2019). Here, we extend the spectrum of molecular methods, and show that unbiased mNGS of single mosquitoes can identify blood meal hosts, while simultaneously validating the mosquito species and providing an unbiased look at the pathogens. This allows for both reservoir identification, which seeks to identify the unknown host of a known pathogen, and xenosurveillance, which seeks to identify the unknown pathogens of specific vertebrate populations (Grubaugh et al., 2015). For example, in this study we found a high prevalence of the tick-borne pathogen Anaplasma in mosquitoes that had likely ingested a blood meal from deer. In one deer-fed mosquito, we found Lobuck virus, a novel orbivirus isolate that belongs to a clade of viruses implicated in a disease of commercially farmed deer reported in Missouri, Florida, and Pennsylvania (Ahasan et al., 2019b, 2019a; Cooper et al., 2014). Our data suggest that mosquito species are a potential vector for such orbiviruses. For these analyses, it was crucial that single mosquitoes were sequenced-if the mosquitoes had been pooled, it would not have been possible to associate potential vertebrate pathogens with a specific blood meal host.

\section{A critical role for public data in public health}

This study would have been impossible without rich public datasets containing sequences, species, locations, and sampling dates. These provided the backbone of information allowing us to identify the majority of our sequences. Citizen scientist resources, such as the iNaturalist catalog of biodiversity observations, was a valuable complement, providing empirical knowledge of species distributions in the mosquito collection area that resolved the ambiguity we detected in sequence space.

In sum, complementing conventional analyses of mosquito pools and field observations of mosquitoes and the animals they bite with single mosquito mNGS can provide valuable complementary information to enhance the evidence base for distinct interventions to control mosquito-borne infectious diseases. As shown here, single mosquito mNGS can map an uncharted landscape related to the movement of pathogens between mosquitoes and their reservoirs. This can inform the deployment of targeted detection or surveillance assays for both established and emerging mosquito-borne pathogens across large geographical areas or animal reservoir populations. As mosquitoes and their microbiota continue to evolve and migrate, posing new risks for human and animal populations, these complementary approaches will empower scientists and public health professionals. 


\section{Data and Code Availability}

Raw and assembled sequencing data are deposited in NCBI Bioproject PRJNA605178. Code is available on Github at https://github.com/czbiohub/california-mosquito-study. Derived data (including all contigs) and supplementary data are available on Figshare at https://doi.org/10.6084/m9.figshare.11832999.v2

\section{Materials and Methods}

\section{Mosquito collection}

Adult mosquitoes were collected at sites indicated in using encephalitis virus survey (EVS) or gravid traps that were baited with $\mathrm{CO}_{2}$ or hay-infused water, respectively. The collected mosquitoes were frozen using dry ice or paralyzed using triethyl amine and placed on a $-15^{\circ} \mathrm{C}$ chill table or in a glass dish, respectively, for identification to species using a dissection microscope. Identified female mosquitoes were immediately frozen using dry ice in deep well 96-well plates and stored at $-80^{\circ} \mathrm{C}$ or on dry ice until the nucleic acids were extracted for sequencing

\section{RNA Preparation}

Individual mosquitoes were homogenized in bashing tubes with 200uL DNA/RNA Shield (Zymo Research Corp., Irvine, CA, USA) using a $5 \mathrm{~mm}$ stainless steel bead and a Tissuelyserll (Qiagen, Valencia, CA, USA) (2x1min, rest on ice in between). Homogenates were centrifuged at $10,000 \mathrm{xg}$ for $5 \mathrm{~min}$ at $4^{\circ} \mathrm{C}$, supernatants were removed and further centrifuged at 16,000xg for $2 \mathrm{~min}$ at $4^{\circ} \mathrm{C}$ after which the supernatants were completely exhausted in the nucleic acid extraction process. RNA and DNA were extracted from the mosquito supernatants using the ZR-DuetTM DNA/RNA MiniPrep kit (Zymo Research Corp., Irvine, CA, USA) with a scaled down version of the manufacturer's protocol with Dnase treatment of RNA using either the kit's DNase or the Qiagen RNase-Free DNase Set (Qiagen, Valencia, CA, USA). Water controls were performed with each extraction batch. Quantitation and quality assessment of RNA was done by the Invitrogen Qubit 3.0 Fluorometer using the Qubit RNA HS Assay Kit (ThermoFisher Scientific, Carlsbad, CA, USA) and the Agilent 2100 BioAnalyzer with the RNA 6000 Pico Kit (Agilent Technologies, Santa Clara, CA, USA).

\section{Library Prep and Sequencing}

Up to 200ng of RNA per mosquito, or 4uL aliquots of water controls extracted in parallel with mosquitoes, were used as input into the library preparation. A 25pg aliquot of External RNA Controls Consortium (ERCC) RNA Spike-In Mix (Ambion, ThermoFisher Scientific, Carlsbad, CA, USA) was added to each sample. The NEBNext Directional RNA Library Prep Kit (Purified mRNA or rRNA Depleted RNA protocol; New England BioLabs, Beverly, MA, USA) and TruSeq Index PCR Primer barcodes (Illumina, San Diego, CA, USA) were used to prepare and index each individual library. The quality and quantity of resulting individual and pooled mNGS libraries were assessed via electrophoresis with the High Sensitivity NGS Fragment Analysis Kit on a Fragment Analyzer (Advanced Analytical Technologies, Inc.), the High-Sensitivity DNA Kit on the Agilent Bioanalyzer (Agilent Technologies, Santa Clara, CA, USA), and via real-time quantitative polymerase chain reaction (qPCR) with the KAPA Library Quantification Kit 
(Kapa Biosystems, Wilmington, MA, USA). Final library pools were spiked with a non-indexed PhiX control library (Illumina, San Diego, CA, USA). Pair-end sequencing ( 2 x 150bp) was performed using an Illumina NovaSeq or Illumina NextSeq sequencing systems (Illumina, San Diego, CA, USA). The pipeline used to separate the sequencing output into 150-base-pair pair-end read FASTQ files by library and to load files onto an Amazon Web Service (AWS) S3 bucket is available on GitHub at https://github.com/czbiohub/utilities.

\section{Mosquito Species Validation}

To validate and correct the visual assignment of mosquito species, we estimated SNP distances between each pair of mosquito transcriptomes by applying SKA (Split Kmer Analysis) (Harris, 2018) to the raw fastq files for each sample. The hierarchical clustering of samples based on the resulting distances was largely consistent with the visual assignments, with each cluster containing a majority of a single species. To correct likely errors in the visual assignment, samples were reassigned to the majority species in their cluster, resulting in 7 changes out of 148 samples and one species assignment for a sample lacking a visual assignment.

\section{Host and Quality Filtering}

Raw sequencing reads were host- and quality-filtered and assembled using the IDseq (v3.2) (Kalantar et al., 2020) platform https://idseq. net, a cloud-based, open-source bioinformatics platform designed for detection of microbes from metagenomic data.

\section{Host Reference}

We compiled a custom mosquito host reference database made up of:

1. All available mosquito genome assemblies under NCBI taxid 7157 (Culicidae; $\mathrm{n}=41$ records corresponding to 28 unique mosquito species, including 1 Culex, 2 Aedes, and 25 Anopheles records) from NCBI Genome Assemblies (accession date: 12/7/2018).

2. All mosquito mitochondrial genome records under NCBI taxid 7157 available in NCBI Genomes (accession date: 12/7/2018; $\mathrm{n}=65$ records).

3. A Drosophila melanogaster genome (GenBank GCF_000001215.4; accession date: 12/7/2018).

Mosquito Genome Assembly and mitochondrial genome accession numbers and descriptions are detailed in Supplemental Data file mosquito_genome_refs.txt. 


\section{Read Filtering}

To select reads for assembly, we performed a series of filtering steps using the IDSeq platform:

\section{Filter Host 1}

Remove reads that align to the host reference using the Spliced Transcripts Alignment to a Reference (STAR) algorithm.

\section{Trim Adapters}

Trim Illumina adapters using trimmomatic.

\section{Quality Filter}

Remove low-quality reads using PriceSeqFilter.

\section{Remove Duplicate Reads}

Remove duplicate reads using CD-HIT-DUP.

\section{Low-Complexity Filter}

Remove low-complexity reads using the LZW-compression filter.

\section{Filter Host 2}

Remove further reads that align to the host reference using Bowtie2, with flag very-sensitive-local.

The remaining reads are referred to as "putative non-host reads." A detailed description of all parameters is available in the IDseq documentation.

https://github.com/chanzuckerberg/idseq-dag/wiki/IDseq-Pipeline-Stage-\%231:-Host-Filtering-and-QC

\section{Assembly}

The putative non-host reads for each sample were assembled into contigs using SPADES (Bankevich et al., 2012) with default settings. The reads used for assembly were mapped back to the contigs using Bowtie2 (Langmead and Salzberg, 2012) (flag very-sensitive), and contigs with more than 2 reads were retained for further analysis.

\section{Taxonomic Assignment}

We aligned each contig to the $n t$ and $n r$ databases using BLASTn (Altschul et al., 1990) (discontinuous megablast) and PLAST (a faster implementation of the BLASTx algorithm), respectively. (The databases were downloaded from NCBI on Mar 27, 2019.) Default parameters were used, except the E-value cutoff was set to 1e-2. For each contig, the results from the database with a better top hit (as judged by bitscore) are used for further analysis.

For contigs with BLAST hits to more than one species, we report the lowest common ancestor (LCA) of all hits whose number of matching aligned bases alignment length*percent identity is no less than the number of aligned bases for the best BLAST hit minus the number of mismatches in the best hit. (In the 
case that the same segment of the query is aligned for all hits, this condition guarantees that the excluded hits are further from the best hit than the query is.)

For 172,244 contigs there were strong BLAST hits to Hexapoda, the subphylum of arthropods containing mosquitoes. This is likely a consequence of the limited number and quality of genomes used in host filtering, and all contigs with an alignment to Hexapoda of at least $80 \%$ of the query length or whose top hit (by e-value) was to Hexapoda were discarded from further analysis.

Contigs with no BLAST hits are referred to as "dark contigs".

For RNA viruses, where complete or near-complete genomes were recovered, a more sensitive analysis was performed.

\section{Viral Polymerase Detection and Segment Assignment}

Alignments of viral RNA-dependent RNA polymerases used to detect domains were downloaded from Pfam. These were RdRP_1 (PF00680, Picornavirales-like and Nidovirales-like), RdRP_2 (PF00978, Tymovirales-like and Hepe-Virga-like), RdRP_3 (PF00998, Tombusviridae-like and Nodaviridae-like), RdRP_4 (PF02123, Toti-, Luteo-, and Sobemoviridae-like), RdRP_5 (PF07925, Reoviridae-like), Birna_RdRp (PF04197, Birnaviridae-like), Flavi_NS5 (PF00972, Flaviviridae-like), Mitovir_RNA_pol (PF05919, Narnaviridae-like), Bunya_RdRp (PF04196, Bunyavirales-like), Arena_RNA_pol (PF06317, Arenaviridae-like), Mononeg_RNA_pol (PF00946, Mononega- and Chuviridae-like), Flu_PB1 (PF00602, Orthomyxoviridae-like). Hidden Markov model (HMM) profiles were generated from these with HMMER (v3.1b2; http://hmmer.org/) and tested against a set of diverged viruses, including ones thought to represent new families. Based on these results only the RdRP_5 HMM was unable to detect diverged Reovirus RdRp, such as Chiqui virus. An additional alternative Reovirus HMM (HMMbuild command) was generated by using BLASTp hits to Chiqui virus, largely to genera Cypovirus and Oryzavirus, aligned with MAFFT (Katoh et al., 2005) (E-INS-i, BLOSUM30).

All contigs of length $>500$ base pairs were grouped into clusters using a threshold of $\geq 99 \%$ identity (CD-HIT-EST (Li and Godzik, 2006)). Representative contigs from each cluster were scanned for open reading frames (standard genetic code) coding for proteins at least 200 amino acids long, in all six frames with a Python script using Biopython (Cock et al., 2009). These proteins were scanned using HMM profiles built earlier and potential RdRp-bearing contigs were marked for follow up. We chose to classify our contigs by focusing on RdRp under the assumption that bona fide exogenous viruses should at the very least carry an RdRp and be mostly coding-complete. Contigs that were not associated with an RdRp or coding-complete included Cell fusing agent virus (Flaviviridae, heavily fragmented) and Phasma-like nucleoprotein sequences (potential piRNAs) in a few samples.

\section{Co-Occurrence}

For each cluster whose representative contig contained a potential RdRp, we identified its putative viral segment from CD-HIT clusters whose set of samples overlapped the set of samples in the RdRp cluster at a threshold of $80 \%$. (That is, a putative segment should be present in at least $80 \%$ of the samples that $\mathrm{RdRp}$ is present in, and $\mathrm{RdRp}$ should be present in at least $80 \%$ of the samples that the putative segment is present in). 
In cases where a singleton segmented (bunya-, orthomyxo-, reo-, chryso-like, etc) virus was detected in a sample we relied on the presence of BLASTx hits of other segments to related viruses (e.g. diverged orthobunyavirus). We thus linked large numbers of viral or likely viral contigs to RdRps representing putative genomes for these lineages.

\section{Final Classification}

There were 1269 contigs identified as viral either by RdRp detection or co-occurrence, and the resulting species-level calls are used for further analysis in lieu of the LCA computed via BLAST alignments. This included 338 "dark contigs" which had no BLAST hits, 748 with LCA in Viruses; the LCAs for the remainder were Bacteria (9), and Eukaryota (4), and Ambiguous (170), a category including (including root, cellular organisms, and synthetic constructs). Reads are assigned the taxonomic group of the contig they map to.

\section{Water Controls and Contamination}

There are many potential sources of contaminating nucleic acid, including lab surfaces, human experimenters, and reagent kits. We attempt to quantify and correct for this contamination using 8 water controls. We model contamination as a random process, where the mass of a contaminant taxon $t$ in any sample (water or Mosquito) is a random variable $X_{t}$. We convert from units of reads to units of mass using the number of ERCC reads for each sample (as a fixed volume of ERCC spike-in solution was added to each sample well). We estimate the mean of $X_{t}$ using the water controls. We say that a taxon observed in a sample is a possible contaminant if the estimated mass of that taxon in that sample is less than 100 times the average estimated mass of that taxon in the water samples. Since the probability that a non-negative random variable is greater than 100 times its mean is at most 1\% (Markov's inequality), this gives a false discovery rate of $1 \%$. For each possible contaminant taxon in a sample, all contigs (and reads) assigned to that taxon in that sample were excluded from further analysis. A total of 46,603 reads were removed as possible contamination using this scheme. (Human and mouse were identified as the most abundant contaminant species.)

For every sample, "classified non-host reads" refer to those reads mapping to contigs that pass the above filtering, Hexapoda exclusion, and decontamination steps. "Non-host reads" refers to the classified non-host reads plus the reads passing host filtering which failed to assemble into contigs or assembled into a contig with only two reads.

\section{Treemap}

Treemaps (e.g. Figure 2) are a way of visualizing hierarchical information as nested rectangles whose area represents numerical values. To visualize the distribution of reads amongst taxonomic ranks, we first split the data into two categories: viral and cellular. For cellular taxonomic ranks (Bacteria, Eukaryotes, Archaea and their descendants) we assigned all reads of a contig to the taxonomic compartment the contig was assigned (see above, "Taxonomic Assignment"). For viral taxa we relied on the curated set of viral contigs coding for RdRp and their putative segments, where a putative taxonomic rank (usually family level) had been assigned. All the reads belonging to contigs that comprised putative 
genomes were assigned to their own compartment in the treemap, under the curated rank. Additional compartments were introduced to either reflect aspects of the outdated and potentially non-monophyletic taxonomy which is nevertheless informative (e.g. positive- or double-strandedness of RNA viruses) or represent previously reported groups without an official taxonomic ID on public databases (e.g. Narna-Levi, Toti-Chryso, Hepe-Virga, etc).

To prototype the cellular part of the treemap, all taxonomic IDs encountered along the path from the assigned taxonomic ID up to root (i.e. the taxonomic ID's lineage) were added to the treemap. Based on concentrations of reads in particular parts of the resulting taxonomic treemap, prior beliefs about the specificity of BLAST hits, and information utility, this was narrowed down to the following taxonomic ranks: Bacteria, Wolbachia, Gammaproteobacteria, Spirochaetes, Terrabacteria group, Fungi, Boroeutheria, Aves, Trypanosomatidae, Leishmaniinae, Viridiplantae.

\section{Microbiota distribution in single mosquitoes}

In Figure 3, the denominators are non-host reads. The numerators are numbers of reads from contigs with confident assignments. For viruses, these contigs came from viral curation or co-occurrence. For Wolbachia and eukaryotes, these contigs had LCA assignment within the Wolbachieae tribe (taxid: 952) and Eukaryota superkingdom (taxid: 2759), respectively, and had a BLAST alignment where the percentage of aligned bases was at least $90 \%$. Groups within viruses, Wolbachia, and eukaryotes were excluded for a given sample if the cumulative proportion of non-host reads was less than $1 \%$. Samples were excluded if the total proportions of non-host reads belonging to viruses, Wolbachia, or eukaryotes were all below $1 \%$.

\section{Blood meal calling}

For each of the 60 blood fed mosquito samples from Alameda County, we selected each contig with LCA in the subphylum Vertebrata, excluding those contained in the order Primates (because of the possibility of contamination with human DNA). For each sample, we identified the lowest rank taxonomic group compatible with the LCAs of the selected contigs. (A taxonomic group is compatible with a set of taxonomic groups if it is an ancestor or descendent of each group in the set.) For 44 of the 45 samples containing vertebrate contigs, this rank is at class or below; for 12 samples, it is at the species level. Each taxonomic assignment falls into one of the following categories: Pecora, Aves, Carnivora, Rodentia, Leporidae. In Figure 5, each sample with a blood meal detected is displayed according to which of those categories it belongs (Underlying data for figure 5 are provided in Supplemental Data Files 12 - 14). The remaining sample, CMS001_022_Ra_S6, contained three contigs mapping to members of Pecora and a single contig with LCA Euarchontoglires, a superorder of mammals including primates and rodents; we annotate this sample as containing Pecora.

Notably, 19 samples contain at least one contig with LCA in genus Odocoileus and another contig with LCA genus Bos. While the lowest rank compatible taxonomic group is the infraorder Pecora, it is likely that a single species endemic in the sampled area is responsible for all of these sequences. Given the observational data in the region (described in the main text), that species is likely a member of Odocoileus whose genome diverges somewhat from the reference. 


\section{Phylogenetic analyses}

We chose a single Wuhan mosquito virus 6 genome from our study (CMS001_038_Ra_S22) as a reference to assemble by alignment the rest of the genome of strain QN3-6 (from SRA entry SRX833542 as only PB1 was available for this strain) and the two small segments discovered here for Australian segments (from SRA entries SRX2901194, SRX2901185, SRX2901192, SRX2901195, SRX2901187, SRX2901189, and SRX2901190) using Magic-BLAST (Boratyn et al., 2018). Due to much higher coverage in Australian samples, Magic-BLAST detected potential RNA splice sites for the smallest segment (hypothetical 3 ) which would extend the relatively short open reading frame to encompass most of the segment. Sequences of each segment were aligned with MAFFT (Auto setting) and trimmed to coding regions. For hypothetical 3 segment we inserted Ns into the sequence near the RNA splice site to bring the rest of the segment sequence into frame.

PhyML (Guindon et al., 2010) was used to generate maximum likelihood phylogenies under an $\mathrm{HKY}+\Gamma_{4}$ model (Guindon et al., 2010; Hasegawa et al., 1985; Yang, 1994). Each tree was rooted via a least-squares regression of tip dates against divergence from root in TreeTime (Sagulenko et al., 2018). Branches with length 0.0 in each tree (arbitrarily resolved polytomies) were collapsed, and trees untangled and visualized using baltic21 (https://github.com/evogytis/baltic).

To generate the Culex narnavirus 1 tanglegrams, 42 sequences of RdRp and 42 co-occurring Robin segment sequences from our samples and three previously published RdRp sequences (MK628543, KP642119, KP642120) as well as their three corresponding Robin segments assembled from SRA entries (SRR8668667, SRR1706006, SRR1705824, respectively) were aligned with MAFFT and trimmed to just the most conserved open reading frame (as opposed to its complement on the reverse strand). Maximum likelihood phylogenies for both RdRp and Robin segments were generated with PhyML with 100 bootstrap replicates under an $\mathrm{HKY}+\Gamma_{4}$ substitution model. The resulting phylogenies were mid-point rooted, untangled and visualized using baltic21 (https://github.com/evogytis/baltic).

\section{Acknowledgments}

We thank our collaborating partners in the California Mosquito and Vector Control Agency Districts of Alameda, Placer, San Diego, West Valley, and Coachella Valley, who provided all the mosquito specimens and corresponding metadata that made this study possible. We thank Maira Phelps for liaison work with collaborators and in-house specimen management. We thank Rene Sit, Michelle Tan, and Norma Neff of the Chan Zuckerberg Biohub Genomics Platform for supporting all aspects of mNGS sequencing for this study. We thank the IDseq team at the Chan Zuckerberg Initiative for useful discussions and facilitation of analysis over the course of this study. We thank Jack Kamm, Darren J Obbard, and Cristina Tato for useful discussions during the development of this project. We would also like to acknowledge Natalie Whitis and Annie Lo for their contribution to the early phases of the specimen extraction and sequencing library preparation for this project. We thank Sandra Schmid, Bill Burkholder, Cristina Tato, Peter Kim, David Yllanes, and Joe DeRisi for reviewing the manuscript. 


\section{References}

Ahasan MS, Campos Krauer JM, Subramaniam K, Lednicky JA, Loeb JC, Sayler KA, Wisely SM, Waltzek TB. $2019 a$. Complete Genome Sequence of Mobuck Virus Isolated from a Florida White-Tailed Deer (Odocoileus virginianus). Microbiol Resour Announc 8. doi:10.1128/MRA.01324-18

Ahasan MS, Subramaniam K, Campos Krauer JM, Sayler KA, Loeb JC, Goodfriend OF, Barber HM, Stephenson CJ, Popov VL, Charrel RN, Wisely SM, Waltzek TB, Lednicky JA. 2019b. Three New Orbivirus Species Isolated from Farmed White-Tailed Deer (Odocoileus virginianus) in the United States. Viruses 12. doi:10.3390/v12010013

Altschul SF, Gish W, Miller W, Myers EW, Lipman DJ. 1990. Basic local alignment search tool. Journal of Molecular Biology 215:403-410. doi:10.1016/S0022-2836(05)80360-2

Atoni E, Wang Y, Karungu S, Waruhiu C, Zohaib A, Obanda V, Agwanda B, Mutua M, Xia H, Yuan Z. 2018. Metagenomic Virome Analysis of Culex Mosquitoes from Kenya and China. Viruses 10. doi:10.3390/v10010030

Atoni E, Zhao L, Karungu S, Obanda V, Agwanda B, Xia H, Yuan Z. 2019. The discovery and global distribution of novel mosquito-associated viruses in the last decade (2007-2017). Rev Med Virol e2079. doi:10.1002/rmv.2079

Bankevich A, Nurk S, Antipov D, Gurevich AA, Dvorkin M, Kulikov AS, Lesin VM, Nikolenko SI, Pham S, Prjibelski AD, Pyshkin AV, Sirotkin AV, Vyahhi N, Tesler G, Alekseyev MA, Pevzner PA. 2012. SPAdes: a new genome assembly algorithm and its applications to single-cell sequencing. J Comput Biol 19:455-477. doi:10.1089/cmb.2012.0021

Batovska J, Lynch SE, Cogan NOI, Brown K, Darbro JM, Kho EA, Blacket MJ. 2018. Effective mosquito and arbovirus surveillance using metabarcoding. Molecular Ecology Resources 18:32-40. doi:10.1111/1755-0998.12682

Bigot D, Atyame CM, Weill M, Justy F, Herniou EA, Gayral P. 2018. Discovery of Culex pipiens associated tunisia virus: a new ssRNA(+) virus representing a new insect associated virus family. Virus Evol 4:vex040.

doi:10.1093/ve/vex040

Boothe E, Medeiros MCl, Kitron UD, Brawn JD, Ruiz MO, Goldberg TL, Walker ED, Hamer GL. 2015. Identification of Avian and Hemoparasite DNA in Blood-Engorged Abdomens of Culex pipiens (Diptera; Culicidae) from a West Nile Virus Epidemic region in Suburban Chicago, Illinois. J Med Entomol 52:461-468. doi:10.1093/jme/tjv029

Boratyn GM, Thierry-Mieg J, Thierry-Mieg D, Busby B, Madden TL. 2018. Magic-BLAST, an accurate DNA and RNA-seq aligner for long and short reads. bioRxiv 390013. doi:10.1101/390013

Breitwieser FP, Lu J, Salzberg SL. 2019. A review of methods and databases for metagenomic classification and assembly. Briefings in Bioinformatics 20:1125-1136. doi:10.1093/bib/bbx120

Cechová L, Durnová E, Sikutová S, Halouzka J, Nemec M. 2004. Characterization of spirochetal isolates from arthropods collected in South Moravia, Czech Republic, using fatty acid methyl esters analysis. J Chromatogr $B$ Analyt Technol Biomed Life Sci 808:249-54. doi:10.1016/j.jchromb.2004.05.014

Chandler JA, Liu RM, Bennett SN. 2015. RNA shotgun metagenomic sequencing of northern California (USA) mosquitoes uncovers viruses, bacteria, and fungi. Front Microbio/ 6:185. doi:10.3389/fmicb.2015.00185

Charon J, Grigg MJ, Eden J-S, Piera KA, Rana H, William T, Rose K, Davenport MP, Anstey NM, Holmes EC. 2019. Novel RNA viruses associated with Plasmodium vivax in human malaria and Leucocytozoon parasites in avian disease. PLoS Pathog 15:e1008216. doi:10.1371/journal.ppat.1008216

Cock PJA, Antao T, Chang JT, Chapman BA, Cox CJ, Dalke A, Friedberg I, Hamelryck T, Kauff F, Wilczynski B, de Hoon MJL. 2009. Biopython: freely available Python tools for computational molecular biology and bioinformatics. Bioinformatics 25:1422-1423. doi:10.1093/bioinformatics/btp163 
Cooper E, Anbalagan S, Klumper P, Scherba G, Simonson RR, Hause BM. 2014. Mobuck virus genome sequence and phylogenetic analysis: identification of a novel Orbivirus isolated from a white-tailed deer in Missouri, USA. J Gen Virol 95:110-116. doi:10.1099/vir.0.058800-0

Cornel AJ, McAbee RD, Rasgon J, Stanich MA, Scott TW, Coetzee M. 2003. Differences in extent of genetic introgression between sympatric Culex pipiens and Culex quinquefasciatus (Diptera: Culicidae) in California and South Africa. J Med Entomol 40:36-51. doi:10.1603/0022-2585-40.1.36

Darsie R, Ward R. 2016. Identification and Geographical Distribution of the Mosquitoes of North America, North of Mexico. University Press of Florida.

Dinan AM, Lukhovitskaya NI, Olendraite I, Firth AE. 2019. A case for a reverse-frame coding sequence in a group of positive-sense RNA viruses. bioRxiv 664342. doi:10.1101/664342

Duguma D, Hall MW, Smartt CT, Debboun M, Neufeld JD. 2019. Microbiota variations in Culex nigripalpus disease vector mosquito of West Nile virus and Saint Louis Encephalitis from different geographic origins. PeerJ 6. doi:10.7717/peerj.6168

Fauver JR, Grubaugh ND, Krajacich BJ, Weger-Lucarelli J, Lakin SM, Fakoli LS, Bolay FK, Diclaro JW, Dabiré KR, Foy BD, Brackney DE, Ebel GD, Stenglein MD. 2016. West African Anopheles gambiae mosquitoes harbor a taxonomically diverse virome including new insect-specific flaviviruses, mononegaviruses, and totiviruses. Virology 498:288-299. doi:10.1016/j.virol.2016.07.031

Frey KG, Biser T, Hamilton T, Santos CJ, Pimentel G, Mokashi VP, Bishop-Lilly KA. 2016. Bioinformatic Characterization of Mosquito Viromes within the Eastern United States and Puerto Rico: Discovery of Novel Viruses. Evol Bioinform Online 12:1-12. doi:10.4137/EBO.S38518

GBD 2017 Causes of Death Collaborators. 2018. Global, regional, and national age-sex-specific mortality for 282 causes of death in 195 countries and territories, 1980-2017: a systematic analysis for the Global Burden of Disease Study 2017. Lancet 392:1736-1788. doi:10.1016/\$0140-6736(18)32203-7

GBD 2017 DALYs and HALE Collaborators. 2018. Global, regional, and national disability-adjusted life-years (DALYs) for 359 diseases and injuries and healthy life expectancy (HALE) for 195 countries and territories, 1990-2017: a systematic analysis for the Global Burden of Disease Study 2017. Lancet 392:1859-1922.

doi:10.1016/S0140-6736(18)32335-3

GBD 2017 Disease and Injury Incidence and Prevalence Collaborators. 2018. Global, regional, and national incidence, prevalence, and years lived with disability for 354 diseases and injuries for 195 countries and territories, 1990-2017: a systematic analysis for the Global Burden of Disease Study 2017. Lancet 392:1789-1858. doi:10.1016/S0140-6736(18)32279-7

Göertz GP, Miesen P, Overheul GJ, van Rij RP, van Oers MM, Pijlman GP. 2019. Mosquito Small RNA Responses to West Nile and Insect-Specific Virus Infections in Aedes and Culex Mosquito Cells. Viruses 11.

doi:10.3390/v11030271

Grubaugh ND, Sharma S, Krajacich BJ, lii LSF, Bolay FK, li JWD, Johnson WE, Ebel GD, Foy BD, Brackney DE. 2015. Xenosurveillance: A Novel Mosquito-Based Approach for Examining the Human-Pathogen Landscape. PLOS Neglected Tropical Diseases 9:e0003628. doi:10.1371/journal.pntd.0003628

Guindon S, Dufayard J-F, Lefort V, Anisimova M, Hordijk W, Gascuel O. 2010. New algorithms and methods to estimate maximum-likelihood phylogenies: assessing the performance of PhyML 3.0. Syst Biol 59:307-321. doi:10.1093/sysbio/syq010

Harris SR. 2018. SKA: Split Kmer Analysis Toolkit for Bacterial Genomic Epidemiology. bioRxiv 453142. doi:10.1101/453142 
Hasegawa M, Kishino H, Yano T. 1985. Dating of the human-ape splitting by a molecular clock of mitochondrial DNA. J Mol Evol 22:160-174. doi:10.1007/BF02101694

Hillman BI, Cai G. 2013. Chapter Six - The Family Narnaviridae: Simplest of RNA Viruses In: Ghabrial SA, editor. Advances in Virus Research, Mycoviruses. Academic Press. pp. 149-176. doi:10.1016/B978-0-12-394315-6.00006-4

Hofmeister EK. 2011. West Nile virus: North American experience. Integrative Zoology 6:279-289.

Huestis DL, Dao A, Diallo M, Sanogo ZL, Samake D, Yaro AS, Ousman Y, Linton Y-M, Krishna A, Veru L, Krajacich BJ, Faiman R, Florio J, Chapman JW, Reynolds DR, Weetman D, Mitchell R, Donnelly MJ, Talamas E, Chamorro L, Strobach E, Lehmann T. 2019. Windborne long-distance migration of malaria mosquitoes in the Sahel. Nature 574:404-408. doi:10.1038/s41586-019-1622-4

iNaturalist. 2020. iNaturalist Research-grade Observations. Occurrence dataset. doi:10.15468/ab3s5x

Kalantar KL, Carvalho T, de Bourcy CFA, Dimitrov B, Dingle G, Egger R, Han J, Holmes OB, Juan Y-F, King R, Kislyuk A, Mariano M, Reynoso LV, Cruz DR, Sheu J, Tang J, Wang J, Zhang MA, Zhong E, Ahyong V, Lay S, Chea S, Bohl JA, Manning JE, Tato CM, DeRisi JL. 2020. IDseq - An Open Source Cloud-based Pipeline and Analysis Service for Metagenomic Pathogen Detection and Monitoring (preprint). Bioinformatics. doi:10.1101/2020.04.07.030551

Katoh K, Kuma K, Toh H, Miyata T. 2005. MAFFT version 5: improvement in accuracy of multiple sequence alignment. Nucleic Acids Res 33:511-518. doi:10.1093/nar/gki198

Kittayapong P, Baisley KJ, Baimai V, O’Neill SL. 2000. Distribution and diversity of Wolbachia infections in Southeast Asian mosquitoes (Diptera: Culicidae). J Med Entomol 37:340-345. doi:10.1093/jmedent/37.3.340

Kothera L, Nelms B, Savage HM, Reisen WK. 2012. Complexity of the Culex pipiens complex in California. Proc Pap Annu Conf Mosq Vector Control Assoc Calif 80:1-3.

Kraemer MUG, Reiner RC, Brady OJ, Messina JP, Gilbert M, Pigott DM, Yi D, Johnson K, Earl L, Marczak LB, Shirude S, Davis Weaver N, Bisanzio D, Perkins TA, Lai S, Lu X, Jones P, Coelho GE, Carvalho RG, Van Bortel W, Marsboom C, Hendrickx G, Schaffner F, Moore CG, Nax HH, Bengtsson L, Wetter E, Tatem AJ, Brownstein JS, Smith DL, Lambrechts L, Cauchemez S, Linard C, Faria NR, Pybus OG, Scott TW, Liu Q, Yu H, Wint GRW, Hay SI, Golding N. 2019. Past and future spread of the arbovirus vectors Aedes aegypti and Aedes albopictus. Nat Microbiol 4:854-863. doi:10.1038/s41564-019-0376-y

Langmead B, Salzberg SL. 2012. Fast gapped-read alignment with Bowtie 2. Nat Methods 9:357-359. doi:10.1038/nmeth.1923

Li C-X, Shi M, Tian J-H, Lin X-D, Kang Y-J, Chen L-J, Qin X-C, Xu J, Holmes EC, Zhang Y-Z. 2015. Unprecedented genomic diversity of RNA viruses in arthropods reveals the ancestry of negative-sense RNA viruses. eLife 4:e05378. doi:10.7554/eLife.05378

Li W, Godzik A. 2006. Cd-hit: a fast program for clustering and comparing large sets of protein or nucleotide sequences. Bioinformatics 22:1658-1659. doi:10.1093/bioinformatics/btl158

Longdon B, Brockhurst MA, Russell CA, Welch JJ, Jiggins FM. 2014. The evolution and genetics of virus host shifts. PLoS Pathog 10:e1004395. doi:10.1371/journal.ppat.1004395

McAbee RD, Green EN, Holeman J, Christiansen J, Frye N, Dealey K, Mulligan FS, Brault AC, Cornel AJ. 2008. Identification of Culex pipiens complex mosquitoes in a hybrid zone of West Nile virus transmission in Fresno County, California. Am J Trop Med Hyg 78:303-310.

Moreira LA, Iturbe-Ormaetxe I, Jeffery JA, Lu G, Pyke AT, Hedges LM, Rocha BC, Hall-Mendelin S, Day A, Riegler M, Hugo LE, Johnson KN, Kay BH, McGraw EA, van den Hurk AF, Ryan PA, O’Neill SL. 2009. A Wolbachia Symbiont in 
Aedes aegypti Limits Infection with Dengue, Chikungunya, and Plasmodium. Cell 139:1268-1278.

doi:10.1016/j.cell.2009.11.042

Petersen LR, Brault AC, Nasci RS. 2013. West Nile Virus: Review of the Literature. JAMA 310:308-315.

doi:10.1001/jama.2013.8042

Pettersson JH-O, Shi M, Eden J-S, Holmes EC, Hesson JC. 2019. Meta-Transcriptomic Comparison of the RNA Viromes of the Mosquito Vectors Culex pipiens and Culex torrentium in Northern Europe. Viruses 11. doi:10.3390/v11111033

Rasgon JL, Scott TW. 2004. An initial survey for Wolbachia (Rickettsiales: Rickettsiaceae) infections in selected California mosquitoes (Diptera: Culicidae). J Med Entomol 41:255-257. doi:10.1603/0022-2585-41.2.255

RATNASINGHAM S, HEBERT PDN. 2007. bold: The Barcode of Life Data System (http://www.barcodinglife.org). Mol Ecol Notes 7:355-364. doi:10.1111/j.1471-8286.2007.01678.x

Reeves LE, Gillett-Kaufman JL, Kawahara AY, Kaufman PE. 2018. Barcoding blood meals: New vertebrate-specific primer sets for assigning taxonomic identities to host DNA from mosquito blood meals. PLOS Neglected Tropical Diseases 12:e0006767. doi:10.1371/journal.pntd.0006767

Richaud A, Frézal L, Tahan S, Jiang H, Blatter JA, Zhao G, Kaur T, Wang D, Félix M-A. 2019. Vertical transmission in Caenorhabditis nematodes of RNA molecules encoding a viral RNA-dependent RNA polymerase. Proc Natl Acad Sci USA 116:24738-24747. doi:10.1073/pnas.1903903116

Roumpeka DD, Wallace RJ, Escalettes F, Fotheringham I, Watson M. 2017. A Review of Bioinformatics Tools for Bio-Prospecting from Metagenomic Sequence Data. Front Genet 8. doi:10.3389/fgene.2017.00023

Sadeghi M, Altan E, Deng X, Barker CM, Fang Y, Coffey LL, Delwart E. 2018. Virome of > 12 thousand Culex mosquitoes from throughout California. Virology 523:74-88. doi:10.1016/j.virol.2018.07.029

Sagulenko P, Puller V, Neher RA. 2018. TreeTime: Maximum-likelihood phylodynamic analysis. Virus Evol 4. doi:10.1093/ve/vex042 
Shi C, Beller L, Deboutte W, Yinda KC, Delang L, Vega-Rúa A, Failloux A-B, Matthijnssens J. 2019. Stable distinct core eukaryotic viromes in different mosquito species from Guadeloupe, using single mosquito viral metagenomics. Microbiome 7:121. doi:10.1186/s40168-019-0734-2

Shi C, Liu Y, Hu X, Xiong J, Zhang B, Yuan Z. 2015. A metagenomic survey of viral abundance and diversity in mosquitoes from Hubei province. PLOS ONE 10:e0129845. doi:10.1371/journal.pone.0129845

Shi M, Lin X-D, Tian J-H, Chen L-J, Chen X, Li C-X, Qin X-C, Li J, Cao J-P, Eden J-S, Buchmann J, Wang W, Xu J, Holmes EC, Zhang Y-Z. 2016. Redefining the invertebrate RNA virosphere. Nature. doi:10.1038/nature20167

Shi M, Neville P, Nicholson J, Eden J-S, Imrie A, Holmes EC. 2017. High-Resolution Metatranscriptomics Reveals the Ecological Dynamics of Mosquito-Associated RNA Viruses in Western Australia. J Virol 91.

doi:10.1128/JVI.00680-17

Tedrow RE, Rakotomanga T, Nepomichene T, Howes RE, Ratovonjato J, Ratsimbasoa AC, Svenson GJ, Zimmerman PA. 2019. Anopheles mosquito surveillance in Madagascar reveals multiple blood feeding behavior and Plasmodium infection. PLOS Neglected Tropical Diseases 13:e0007176. doi:10.1371/journal.pntd.0007176

Thongsripong P, Chandler JA, Green AB, Kittayapong P, Wilcox BA, Kapan DD, Bennett SN. 2018. Mosquito vector-associated microbiota: Metabarcoding bacteria and eukaryotic symbionts across habitat types in Thailand endemic for dengue and other arthropod-borne diseases. Ecol Evol 8:1352-1368. doi:10.1002/ece3.3676

Tomazatos A, Jansen S, Pfister S, Török E, Maranda I, Horváth C, Keresztes L, Spînu M, Tannich E, Jöst H, Schmidt-Chanasit J, Cadar D, Lühken R. 2019. Ecology of West Nile Virus in the Danube Delta, Romania: Phylogeography, Xenosurveillance and Mosquito Host-Feeding Patterns. Viruses 11:1159. doi:10.3390/v11121159

Werren JH, Baldo L, Clark ME. 2008. Wolbachia: master manipulators of invertebrate biology. Nat Rev Microbiol 6:741-751. doi:10.1038/nrmicro1969

WHO. 2017. Global Vector Control Response 2017-2030.

Wilson AL, Courtenay O, Kelly-Hope LA, Scott TW, Takken W, Torr SJ, Lindsay SW. 2020. The importance of vector control for the control and elimination of vector-borne diseases. PLoS Negl Trop Dis 14:e0007831.

doi:10.1371/journal.pntd.0007831

Xia H, Wang Y, Shi C, Atoni E, Zhao L, Yuan Z. 2018. Comparative Metagenomic Profiling of Viromes Associated with Four Common Mosquito Species in China. Virol Sin 33:59-66. doi:10.1007/s12250-018-0015-4

Xiao P, Han J, Zhang Y, Li C, Guo X, Wen S, Tian M, Li Y, Wang M, Liu H, Ren J, Zhou H, Lu H, Jin N. 2018a. Metagenomic Analysis of Flaviviridae in Mosquito Viromes Isolated From Yunnan Province in China Reveals Genes From Dengue and Zika Viruses. Front Cell Infect Microbiol 8:359. doi:10.3389/fcimb.2018.00359

Xiao P, Li C, Zhang Y, Han J, Guo X, Xie L, Tian M, Li Y, Wang M, Liu H, Ren J, Zhou H, Lu H, Jin N. 2018b. Metagenomic Sequencing From Mosquitoes in China Reveals a Variety of Insect and Human Viruses. Front Cell Infect Microbiol 8:364. doi:10.3389/fcimb.2018.00364

Yang Z. 1994. Maximum likelihood phylogenetic estimation from DNA sequences with variable rates over sites: Approximate methods. J Mol Evol 39:306-314. doi:10.1007/BF00160154 
bioRxiv preprint doi: https://doi.org/10.1101/2020.02.10.942854; this version posted December 21, 2020. The copyright holder for this preprint (which was not certified by peer review) is the author/funder, who has granted bioRxiv a license to display the preprint in perpetuity. It is made available under aCC-BY 4.0 International license.

Zeller HG, Karabatsos N, Calisher ChH, Digoutte J-P, Murphy FA, Shope RE. 1989. Electron microscopy and antigenic studies of uncharacterized viruses. I. Evidence suggesting the placement of viruses in familiesArenaviridae, Paramyxoviridae, orPoxviridae. Archives of Virology 108:191-209. doi:10.1007/BF01310934 
bioRxiv preprint doi: https://doi.org/10.1101/2020.02.10.942854; this version posted December 21, 2020. The copyright holder for this preprint (which was not certified by peer review) is the author/funder, who has granted bioRxiv a license to display the preprint in perpetuity. It is made available under aCC-BY 4.0 International license.

\section{Figures}

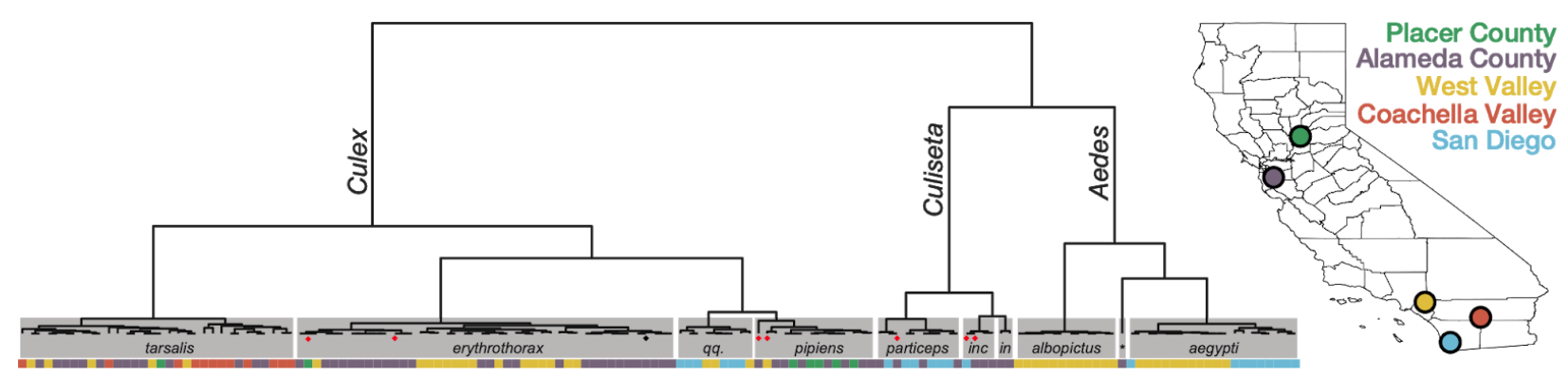

Figure 1. Whole transcriptome analysis for mosquito species identification. Hierarchical clustering of pairwise single-nucleotide polymorphism (SNP) distances between whole transcriptome sequences from the 148 mosquitoes included in this study estimated using SKA (Harris, 2018). The inferred mosquito species for each cluster (text in gray boxes) is the consensus of the species calls made by visual inspection during sample collection for samples in that cluster (qq. = Culex quinquefasciatus, part. = Culiseta particeps, inc $=$ Culiseta incidens, in $=$ Culiseta inornata, albo $=$ Aedes albopictus, ${ }^{*}=$ Aedes dorsalis). Red dots below the nodes on the tree highlight mismatches $(n=7)$ between consensus transcriptome species call and initial visual species call; black dot indicates a sample missing a visual species call. Color bar below the tree shows the collection location for each sample, coded according to the California map legend at right. 


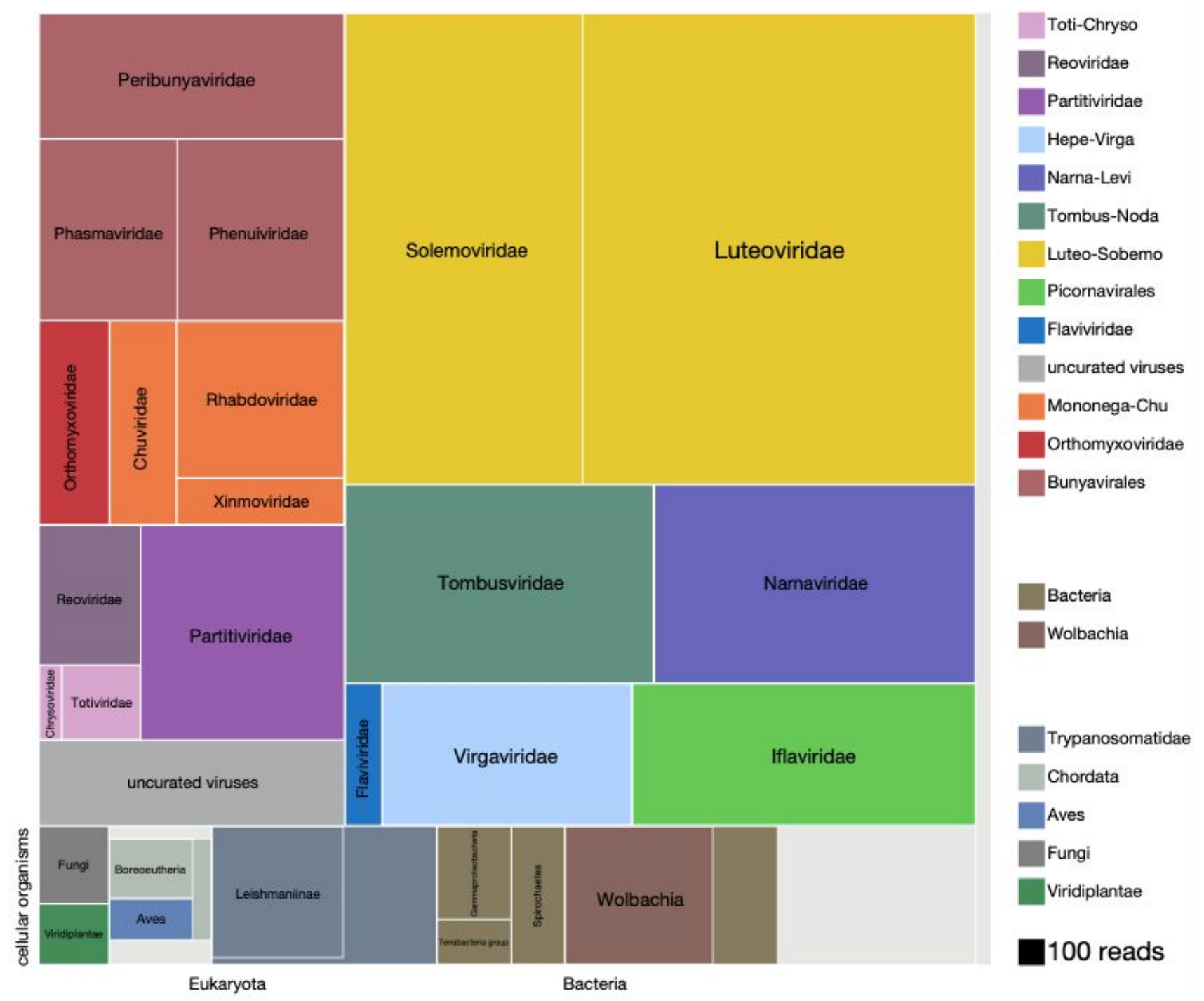

Figure 2. Viruses dominate the microbial signature of mosquitoes. Treemap plot of the proportion of reads recovered among non-host contigs assembled from the 148 single mosquitoes, with viral taxa making up the top portion of the plot above "cellular organisms" label at lower left edge of image, which designates reads assembled into contigs encompassing prokaryotic taxa, the eukaryotic taxa and the taxonomically ambiguous "cellular organisms" that were not possible to assign to a higher resolution (light gray box). The block areas are plotted in proportion to the number of reads assembled into contigs that could be assigned to a given taxa (see area scale, legend). The gray block labeled "uncurated viruses" corresponds to the number of reads assembled into contigs that were clearly viral in origin, but difficult to further resolve due fragmented homology and/or the lack of an associated RNA-dependent RNA polymerase. 


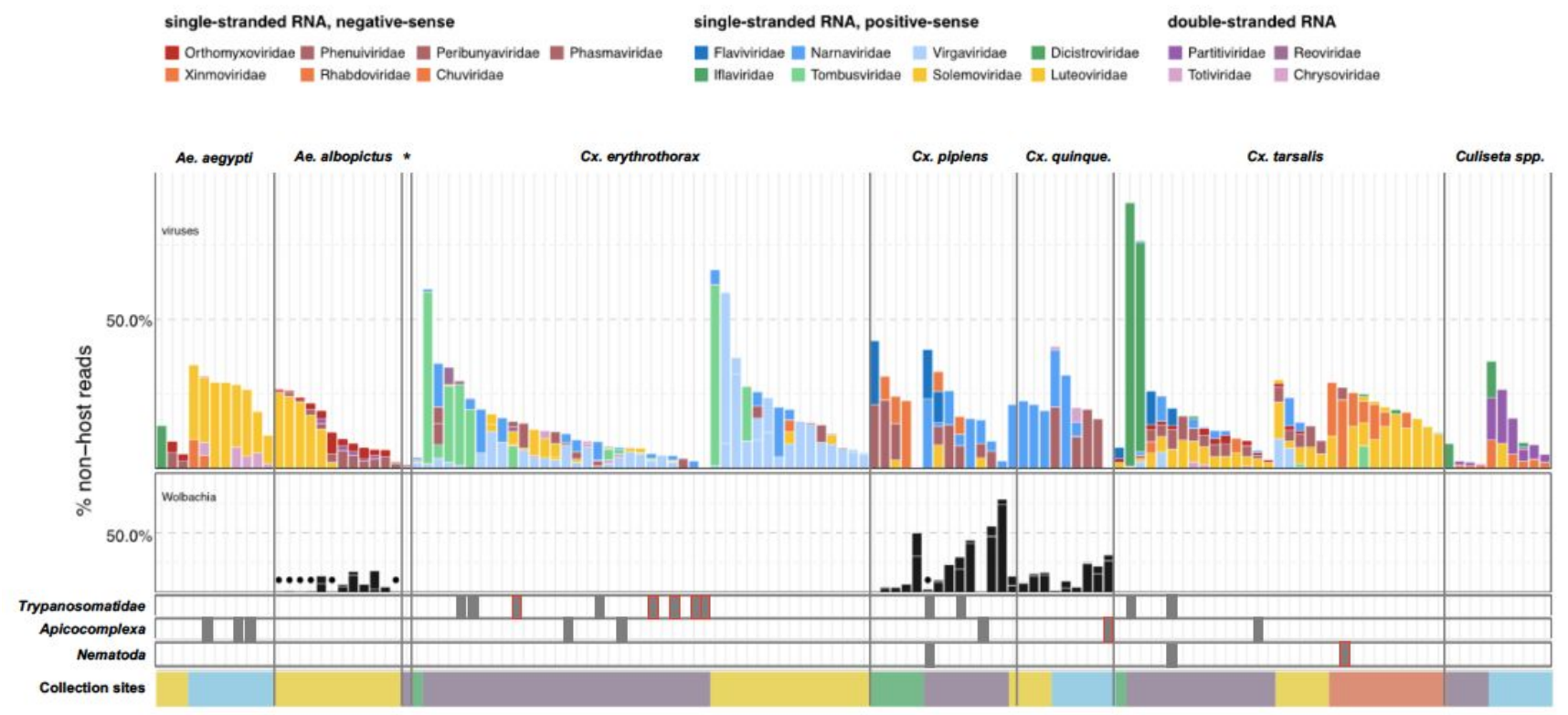

Figure 3. Single mosquito sequencing reveals the heterogeneity of microbiota between individuals, species and locations. Plotted bars report the proportions of non-host reads from individual mosquitoes that are supported by $\geq 1 \%$ of non-host reads corresponding to assembled contigs identified as viruses (top panel), Wolbachia (middle panel), and selected eukaryotic microbes (bottom panel). Species are color coded as indicated in the legend (top of graph). Plotted symbols on the Wolbachia and eukaryotic panels indicate microbes confidently identified, but present at $<1 \%$ of non-host reads (middle panel, black circles = Wolbachia taxa; bottom panels, gray bars are plotted in samples with

Trypanosomatidae, Apicomplexa or Nematoda taxa; gray bars outlined in red indicate detection of these taxa at $\geq 1 \%$ of non-host reads. Samples were clustered by mosquito species (top labels) and ordered by: i) collection site location from north to south (indicated at the bottom, colored as in Figure 1) and ii) viral abundance (descending order, left to right). 
A

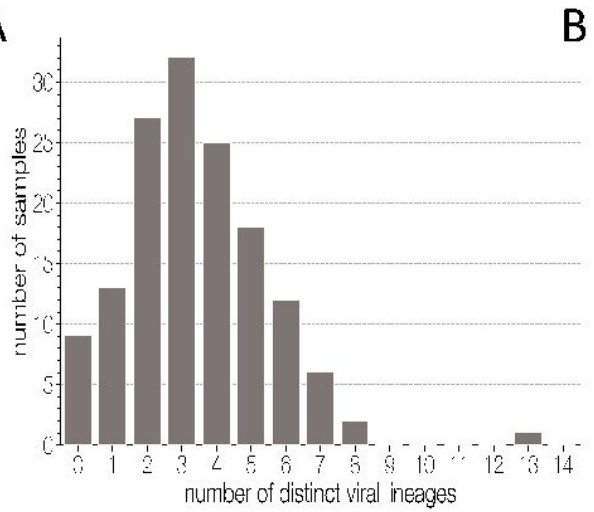

B

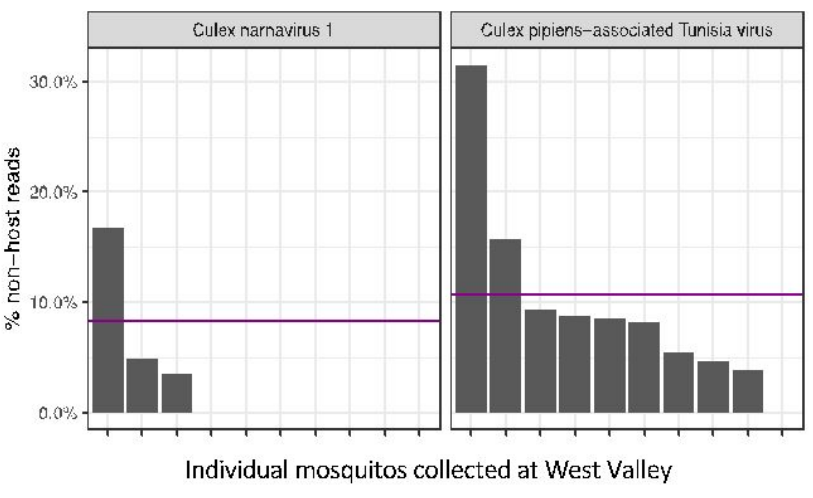

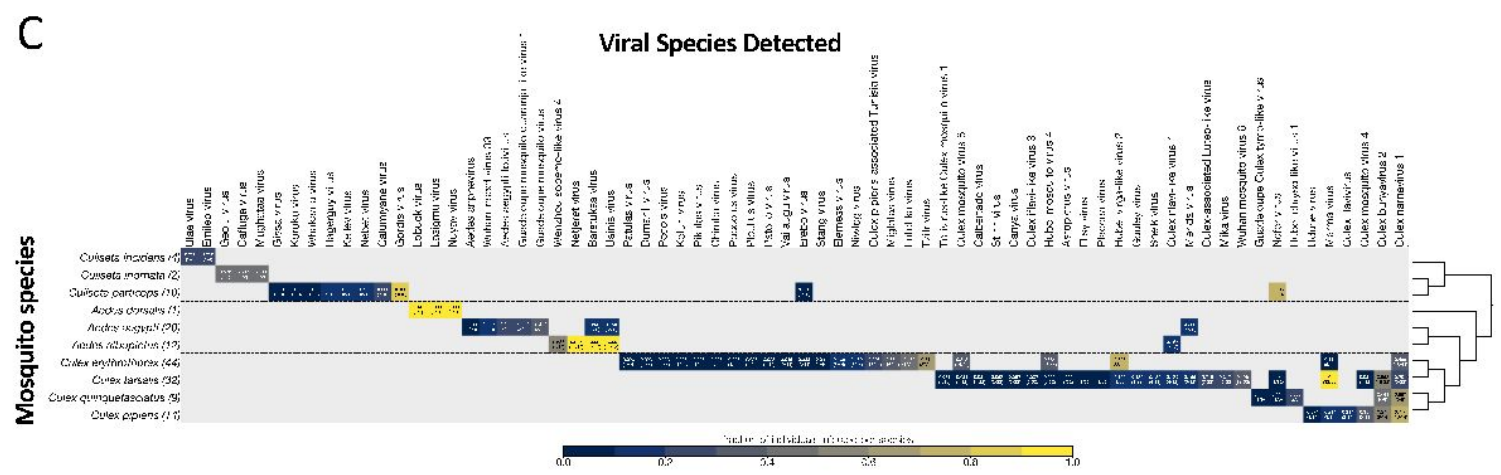

Figure 4. Quantifying viral diversity in and prevalence among single mosquitoes (A) Distribution of mosquitoes within the study in which no, one, or multiple viral lineages were detectable. The sample with 13 distinct viral lineages is sample CMS002_053a that contains six Botourmia-like viruses thought to primarily infect fungi and in which evidence of an ergot fungus was detected. (B) An example of viruses with similar bulk abundance, but different prevalence. Both Culex narnavirus 1 and Culex pipiens-associated Tunisia virus were found among the 10 Culex erythrothorax mosquitoes collected at the same collection site in West Valley. The bulk abundances were calculated by the mean \% non-host reads averaged across the 10 mosquitoes for Culex narnavirus 1 and Culex pipiens-associated Tunisia virus were $8.3 \%$ and $10.6 \%$, respectively (as indicated by the red line). However, the prevalence (i.e. the percent of single mosquitos carrying the virus) was markedly different (C). Global analysis of viral prevalence measured in this study. For each virus, the fraction of individuals infected within each species was calculated, shown on a color scale. Mosquito species arranged according to a phylogeny based on the cytochrome c oxidase subunit I (COI) gene. 


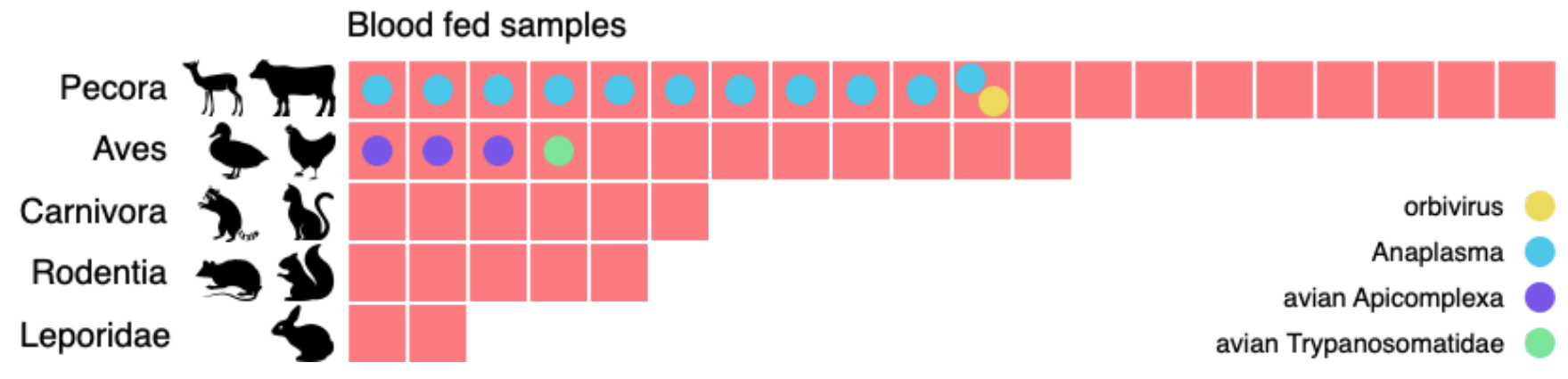

Figure 5. Metagenomic identification of sources of blood meals in individual mosquitos Consensus taxonomic calls of vertebrate contigs for 45 of 60 blood fed mosquitoes collected in Alameda County. The remaining 15 samples had no vertebrate contigs. Red blocks represent individual mosquito samples; colored circles represent co-occurring contigs matching Orbivirus, Anaplasma, Avian Apicomplexa and Avian Trypanosomatidae representing possible bloodborne pathogens of the blood meal host.

A

(1) Identify clusters of homolagous contigs across samples.

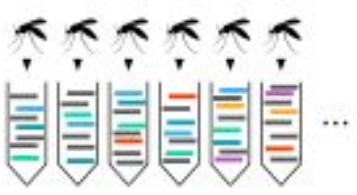

(2) Group clusters by co-occurrence across samples

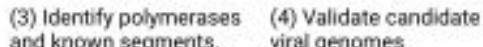

Samples
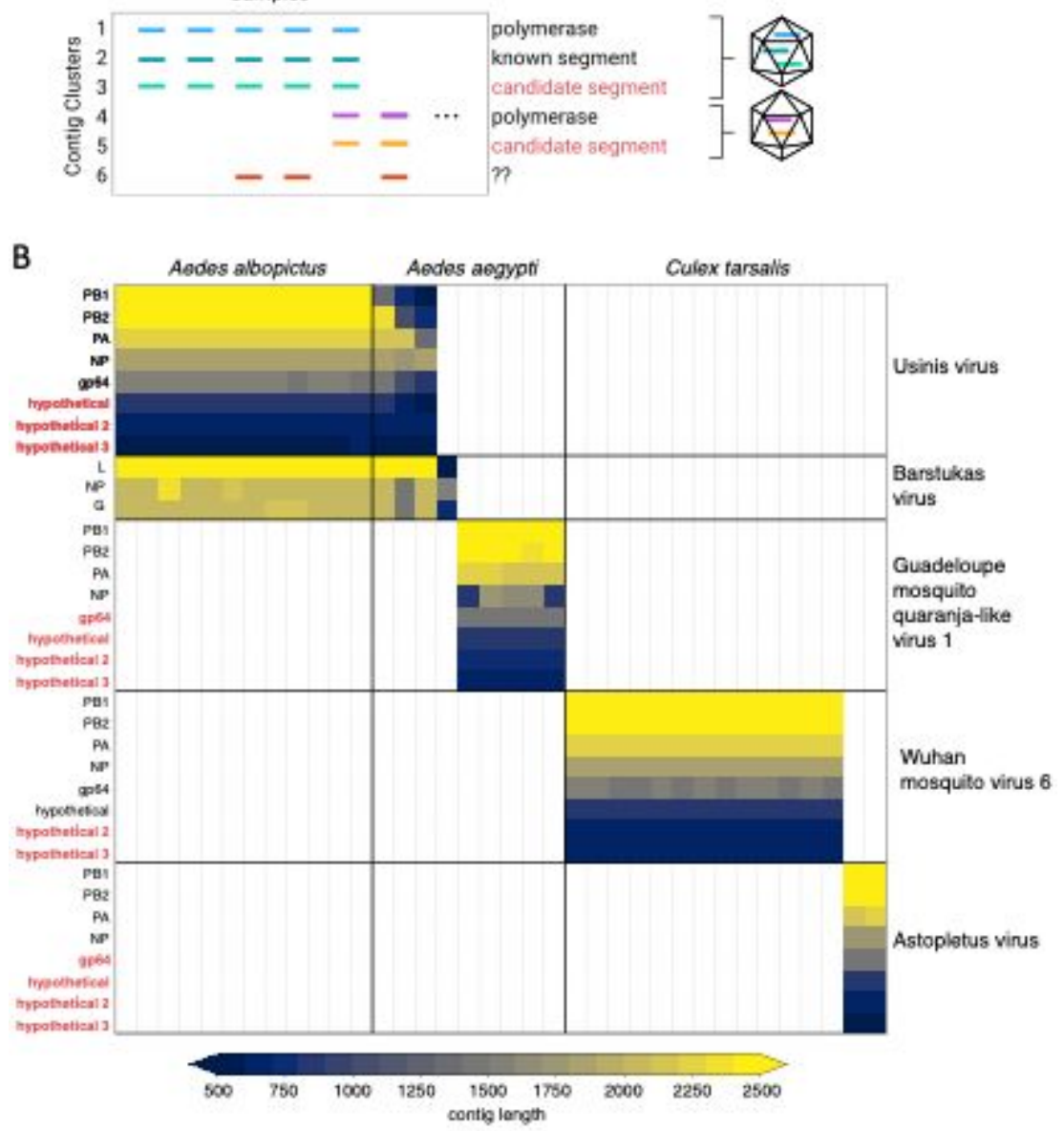
Figure 6. Bringing viral genomic dark matter into the light through single mosquito next generation metagenomic sequencing. (A) Previously unrecognized viral genome segments were identified among unaligned "dark matter" contigs using co-occurrence analysis, which assumes that all segments of a segmented virus will co-occur in the samples where that virus is present and be absent in samples where the virus is absent. (B) Matrix of contigs derived from four distinct Orthomyxoviruses and one Phasma-like virus that were detected via their distinct co-occurrence pattern across mosquitoes. Rows are clusters of highly similar (>99\% identity) contigs and columns are individual mosquito samples. Light grey vertical lines delineate mosquito samples, dark black vertical lines indicate boundaries between mosquito species of each sample. Dark horizontal lines delineate segments comprising viral genomes. Labels on the right indicate viruses, with genomes delineated by horizontal lines. Guadeloupe mosquito quaranja-like virus 1 and Wuhan mosquito virus 6 were previously described and Ūsinis, Barstukas and Astopletus were named here. At left, plain text indicates putative labels for homologous clusters; black text indicates segments identifiable via homology (BLASTx) and red text indicates contig clusters that co-occur with identifiable segments but themselves have no identifiable homology to anything in GenBank. The Phasma-like Barstukas virus exhibits a nearly perfect overlap with Ūsinis virus (except for one sample in which Ūsinis was not found) but is identifiable as a Bunya-like virus due to having a three-segmented genome with recognizable homology across all segments to other Phasma-like viruses. Cells are colored by contig lengths (see color scale legend), highlighting their consistency which is expected of genuine segments. Deviations in detected contig lengths (e.g., Aedes aegypti samples that harbor shorter Ūsinis virus genome segments) reflect the presence of partial or fragmented contig assemblies in some of the samples.

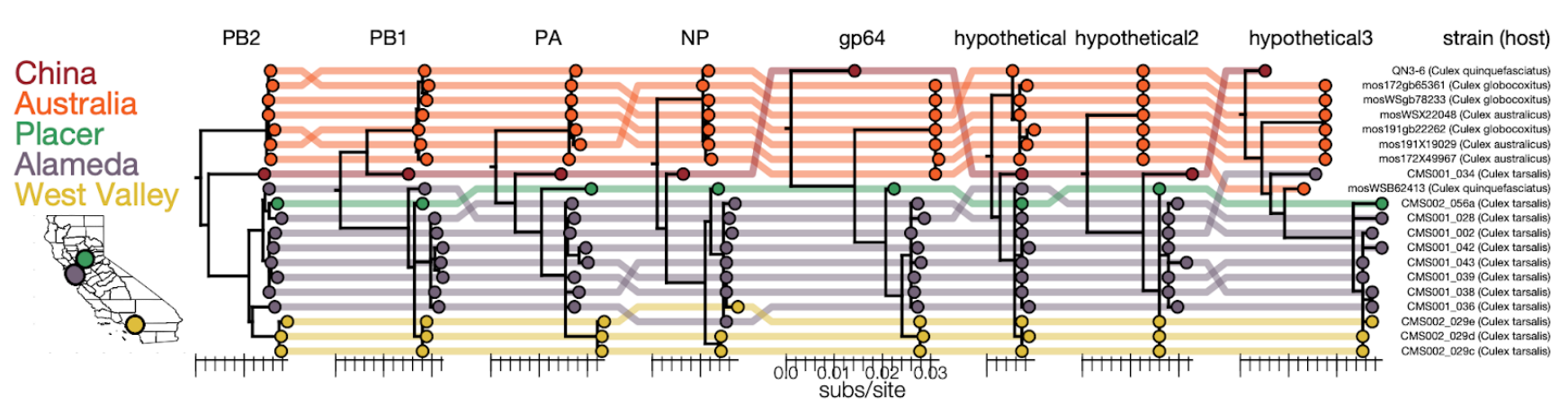

Figure 7. Genomic evidence for segment reassortment and intercontinental spread of mosquito-borne quaranjaviruses (A) The Chinese strain (QN3-6) was originally described from a single PB1 sequence, while Australian (orange) viruses were described as having 6 segments. In this study we report the existence of two additional smaller segments (named hypothetical 2 and hypothetical 3) which we have assembled from our samples and the SRA entries of Chinese and Australian samples. Strains recovered in California as part of this study are colored by sampling location (Placer County in green, Alameda County in purple, West Valley in yellow). Strain names and hosts are indicated on the far right with colored lines tracing the position of each tip in other segment trees with crossings visually indicating potential reassortments. 


\section{Supplemental Figures}

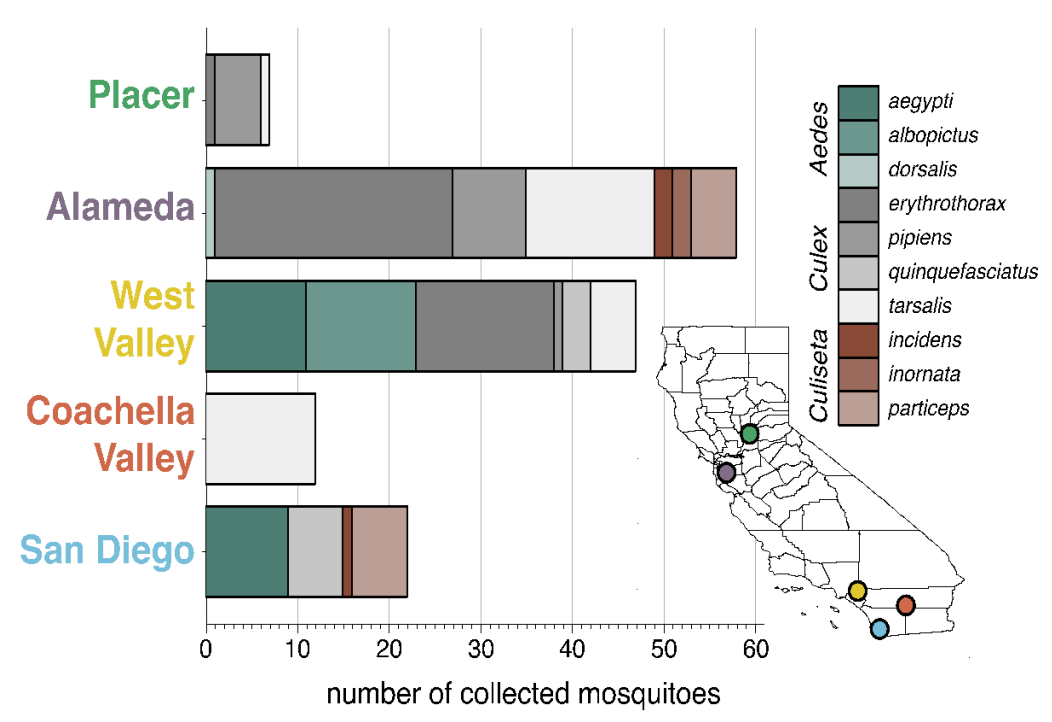

Supplemental Figure 1. Diversity of individual mosquitoes collected across California. Plot shows the number of each genus and species of mosquitoes collected across 5 regions in California: Placer County, Alameda County, West Valley, Coachella Valley, and San Diego, as indicated by the correspondingly colored dots on the map, lower right). Colors in the bar graphs correspond to mosquito species as indicated in the legend. 
bioRxiv preprint doi: https://doi.org/10.1101/2020.02 10.942854; this version posted December 21, 2020. The copyright holder for this preprint (which was not certified by peer review) is the author/funder, who has granted bioRxiv a license to display the preprint in perpetuity. It is made available under aCC-BY 4.0 International license.

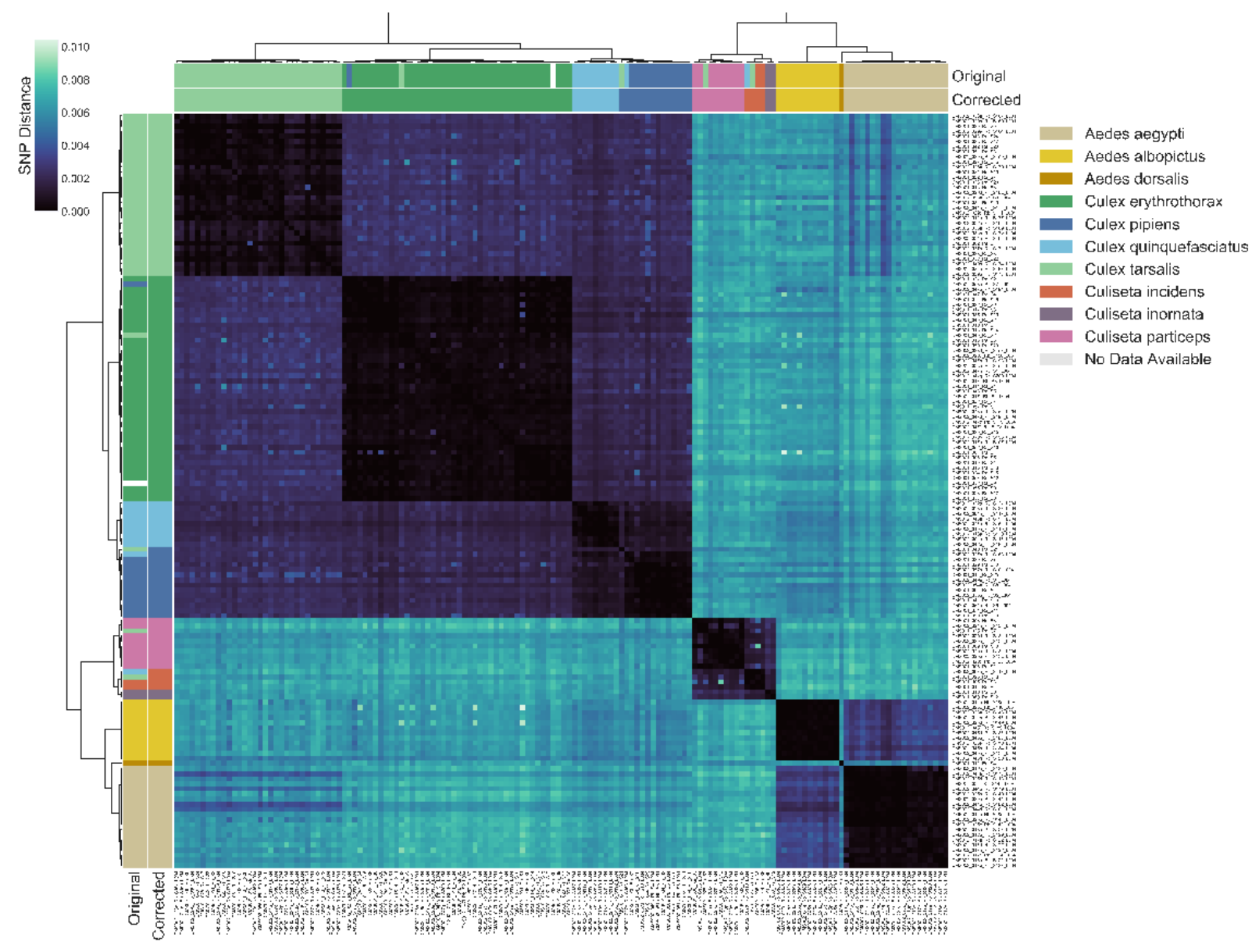

Supplemental Figure 2: Transcriptome-based identification of mosquito species. Hierarchical clustering of pairwise single-nucleotide polymorphism (SNP) distances between samples estimated using SKA (Harris, 2018), ranging from 0 (no SNPs detected, black) to 0.01 ( $1 \%$ of comparable sites had SNPs, blue). Outside color bar indicates visual species label for each sample (Visual), inside color bar indicates consensus transcriptome species label for each sample's cluster (Computed). Computed mosquito species calls were used when Visual and Computed calls were discordant. 


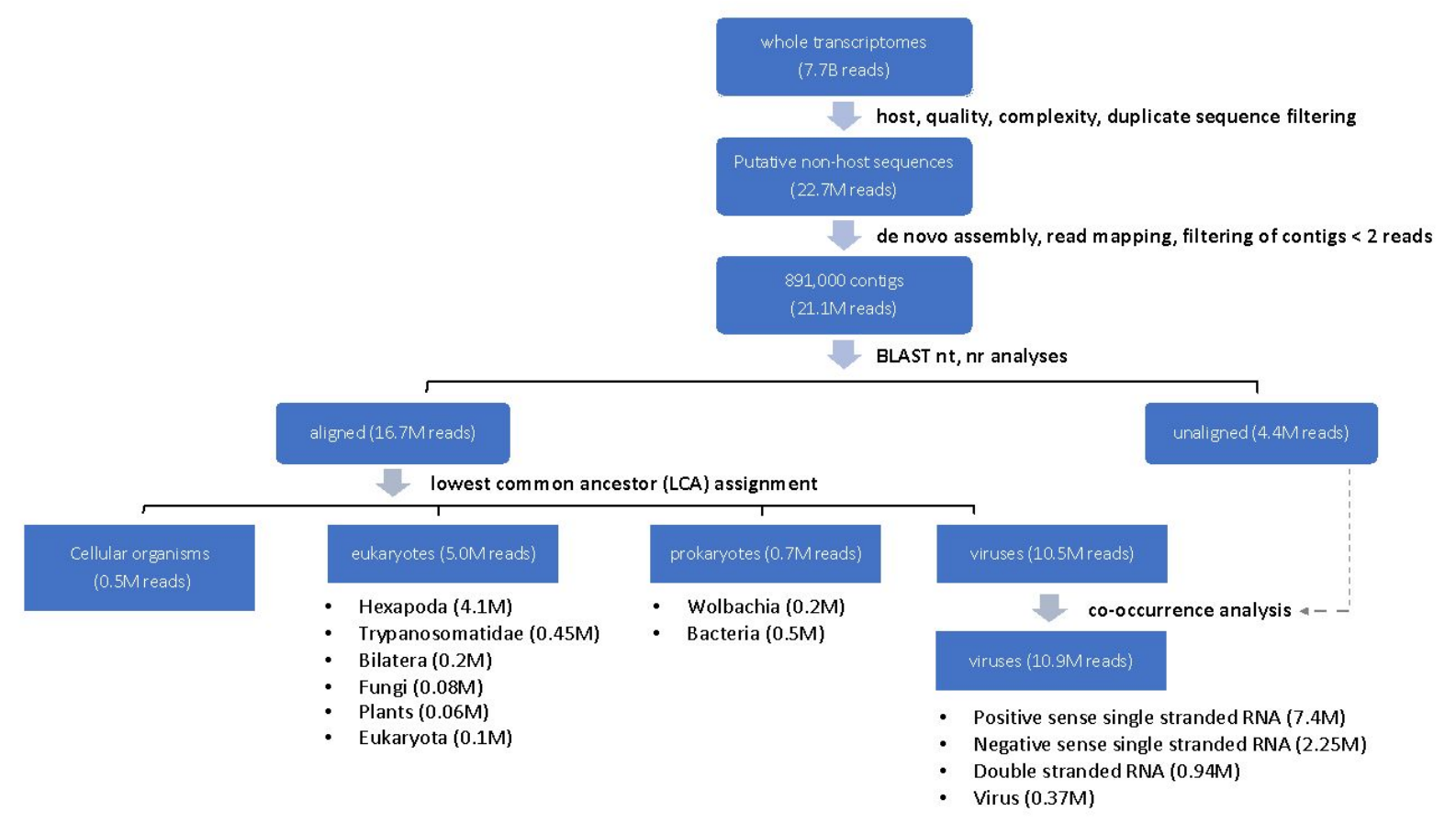

Supplemental Figure 3: Summary of single mosquito mNGS analysis pipeline with sequence recovery yields. A set of 7.78 billion reads served as input transcriptome sequence data for analysis of the taxonomic distribution of 148 single mosquitoes sequenced in this study. We first filtered host, low quality, and low complexity sequences from each transcriptome dataset. A total set of 22.7 million reads passed all of these filters. A set of 891,00 contigs were de novo assembled from 21.1 million of these reads (93\%). To classify and quantify the reads assembled into the contigs into viral, eukaryotic or prokaryotic taxa, we aligned each contig to the NCBI nucleotide and non-redundant protein databases. Recovery of complete or near complete genomes of RNA viruses facilitated viral taxonomic assignment directly from the BLAST results. We further curated contigs likely to code for viral RNA-dependent RNA polymerases (RdRps) and contigs that were physically unlinked but showed evidence of co-occurrence with viral RdRps (labeled "co-occurrence analysis", above; details in Materials and Methods). In contrast, only a fraction of a genome was generally detected among contigs aligning to prokaryotic and eukaryotic taxa. Here, we employed a lowest common ancestor (LCA) analysis of the BLAST results for these contigs (Materials and Methods) to infer their corresponding taxon assignments.

We found that a significant proportion of reads assembled into contigs did not readily align to microbial species or plausible bloodmeal hosts. One set of 4.1 million reads contributed to contigs classified as Hexapoda. These likely correspond to mosquito host sequences too diverged from available mosquito genome reference sequences to be recognized at the host filtering step. As such, these reads were excluded from further analysis. An additional set of 4.4 million reads were assembled into contigs corresponding to metagenomic "dark matter", i.e. contigs without recognizable homology to previously published sequences. Co-occurrence analysis (see main text), allowed us to identify additional viral contigs from this set of contigs, contributing 0.4 million reads to the viral reads tally. This provided a final set of 13.4 million reads assembled into contigs with BLAST alignments to enable analysis of the distribution of viral, bacterial, or eukaryotic taxa detectable among all the mosquitoes analyzed for this study. 
bioRxiv preprint doi: https://doi.org/10.1101/2020.02.10.942854; this version posted December 21, 2020. The copyright holder for this preprint (which was not certified by peer review) is the author/funder, who has granted bioRxiv a license to display the preprint in perpetuity. It is made available under aCC-BY 4.0 International license.

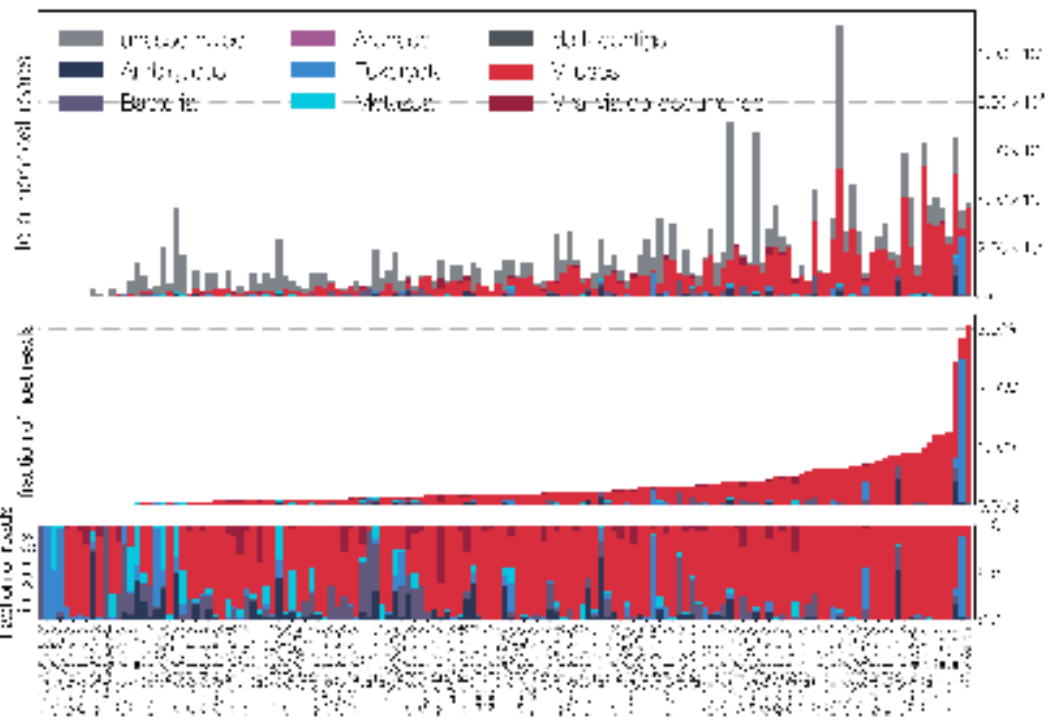

Supplemental Figure 4: Summary of all reads in all sampled mosquitos A breakdown of reads for each sample, into unassembled (those which did not assemble into contigs), dark (those which assembled but were not identifiable taxonomically), Ambiguous (those with LCA at root or cellular organisms), Bacteria, Archaea, Eukaryota, Metazoa, Viruses (via alignment to reference), and Viral via co-occurrence analysis (See Figure 6). Top panel is a stacked bar decomposing the total reads per sample. Middle panel shows the break-down scaled as a fraction of all reads in a sample. Bottom panel shows the fractional breakdown among assembled reads.

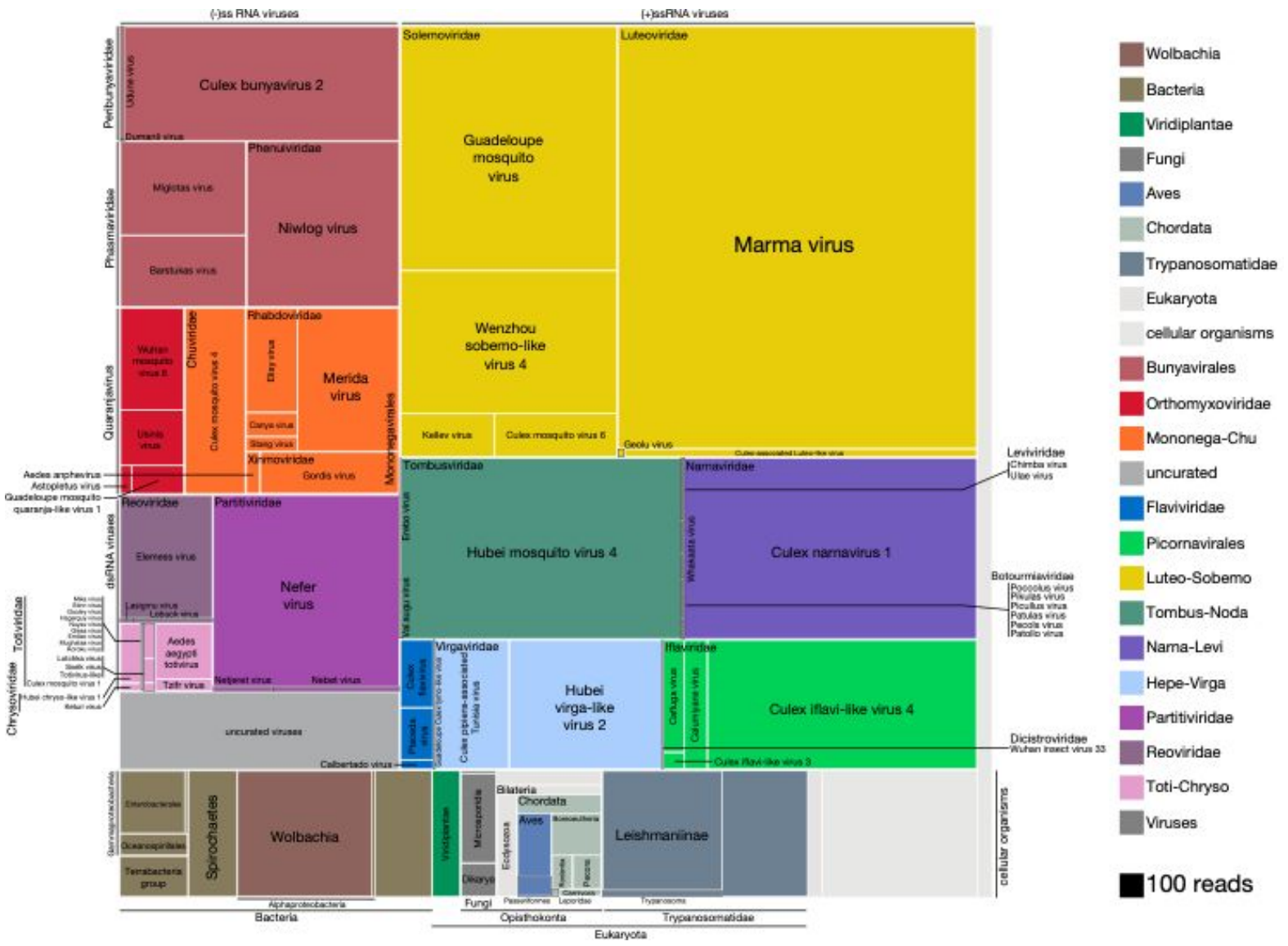

Supplemental Figure 5: High resolution breakdown the microbial signature of mosquitoes. Shown is the read count distribution across non-host taxonomic groups detected among the set of 148 mosquitoes. Treemap overview of taxa detected among the assembled contigs are plotted in squares shaded according to taxon identity (legend) and sized according to number of reads supporting each contig (scaled as indicated, bottom right). Due to the uncertainty in prokaryotic and eukaryotic taxonomic assignments, cellular organisms are assigned to the lowest plausible taxonomic rank. Each distinct viral lineage is displayed as its own compartment. Reddish hues indicate negative-sense RNA viruses; purple hues indicate double-stranded RNA viruses; blue, green and 
bioRxiv preprint doi: https://doi.org/10.1101/2020.02 10.942854; this version posted December 21, 2020. The copyright holder for this preprint (which was not certified by peer review) is the author/funder, who has granted bioRxiv a license to display the preprint in perpetuity. It is made available under aCC-BY 4.0 International license.

yellow hues indicate positive-sense RNA viruses. The "uncurated viruses" box (light grey, lower left) indicates contigs that were clearly viral in origin but too fragmented and/or not associated with an RNA-dependent RNA polymerase.

A

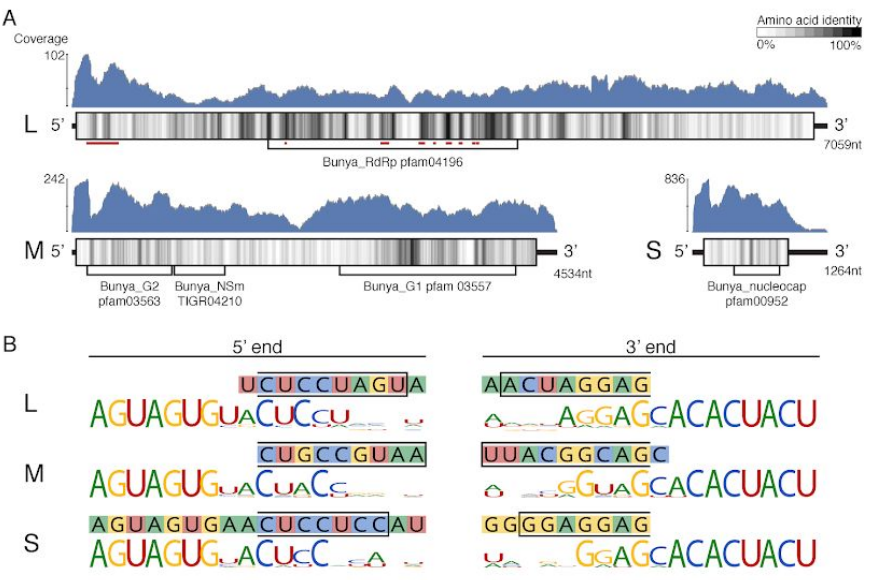

C

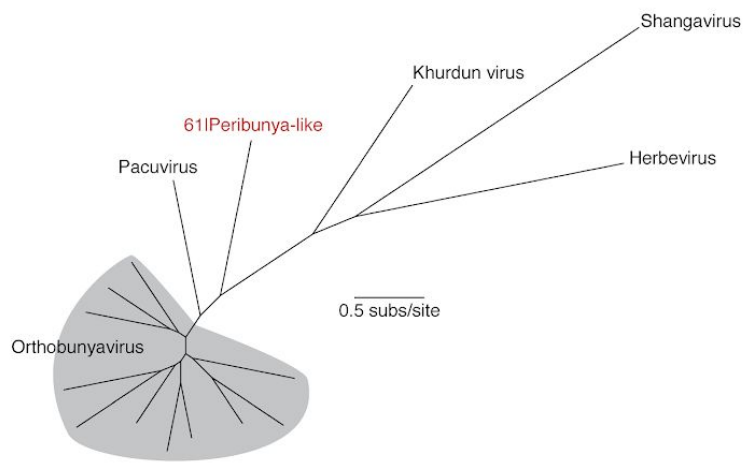

Supplemental Figure 6. Analysis of novel peribunya-like virus showing completeness. (A) Read coverage in blue across the length of each genome segment. Diagrams of genome segments display the longest ORF and indicate amino acid identity with a sliding window of 15 amino acids. Regions of homology to known domains are indicated by Pfam labels, and on the $L$ segment, the locations of canonical RdRp motifs are indicated by red underlining. (B) Ends of the assembled contigs are shown (sequence in 1lled boxes). Regions complementary between $5^{\prime}$ and $3^{\prime}$ end for each segment are indicated by a black outline. These contig ends were aligned to the conserved end sequences of orthobunya and nearby peribunya viruses (logo diagram shows conservation after alignment of reference sequences). (C) Maximum likelihood phylogenetic tree with representative orthobunyaviruses and nearby peribunyaviruse

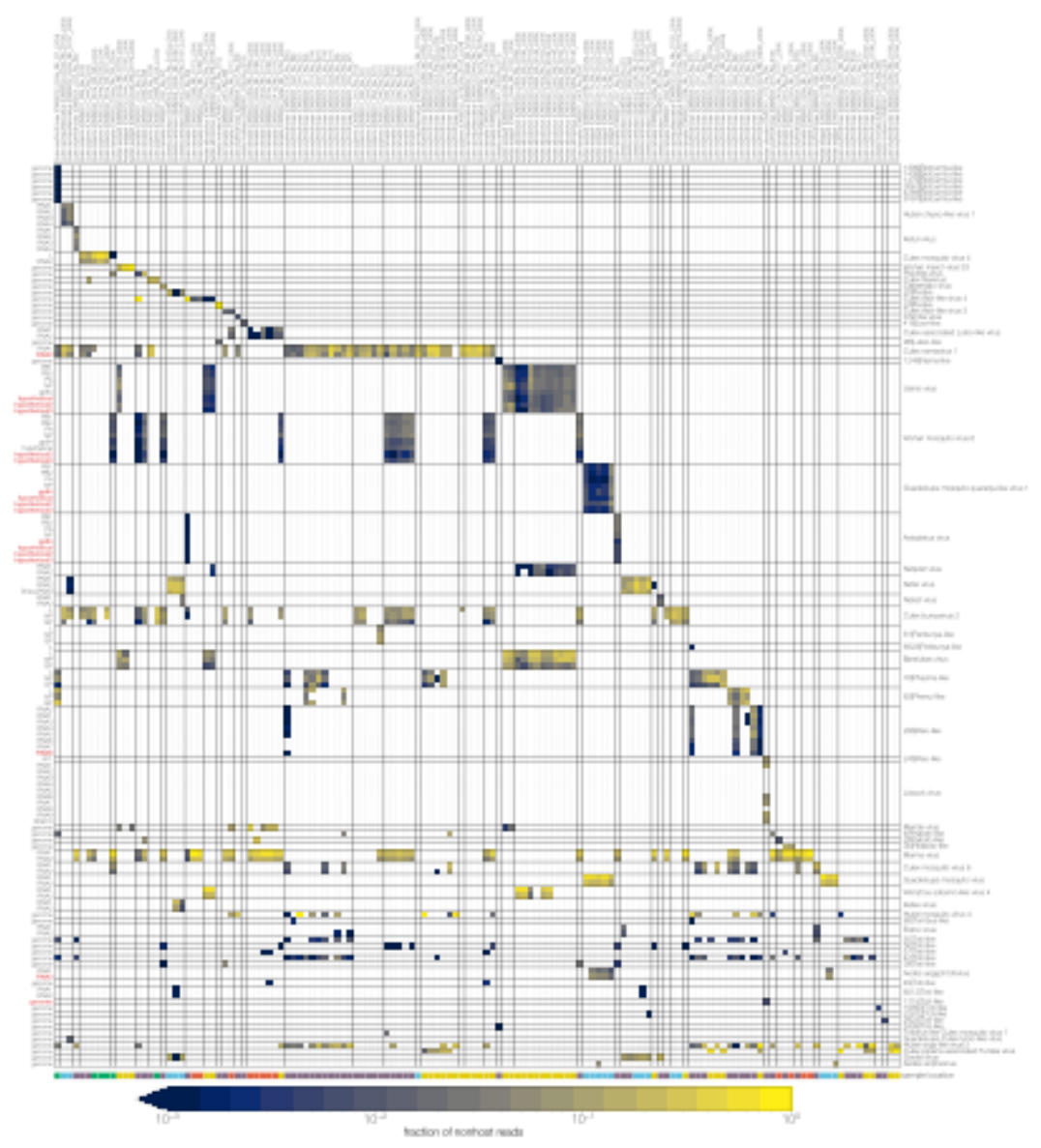

Supplemental Figure 7. Identifying novel RNA segments in the 'dark matter' by co-occurrence. Rows indicate groups of contigs clustered based on nucleotide identity (>99\%). Columns are samples with computed mosquito species indicated. Cells are colored by the fraction of non-host reads in the sample with color bar legend at the bottom. Clusters of rows likely comprising a single genome are separated from other such putative genomes by black horizontal lines with identified or proposed virus names. Black vertical lines delineate blocks of neighboring samples with the same computed mosquito species. Sampling locations are indicated by colored rectangles at the bottom, above the color bar legend. 
bioRxiv preprint doi: https://doi.org/10.1101/2020.02.10.942854; this version posted December 21, 2020. The copyright holder for this preprint (which was not certified by peer review) is the author/funder, who has granted bioRxiv a license to display the preprint in perpetuity. It is made available under aCC-BY 4.0 International license.

A) gp64

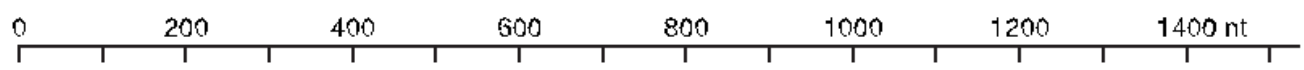

1170 I WMV6

1168 | Osinis

1160 | Astopletus

1181 I GMQLV1

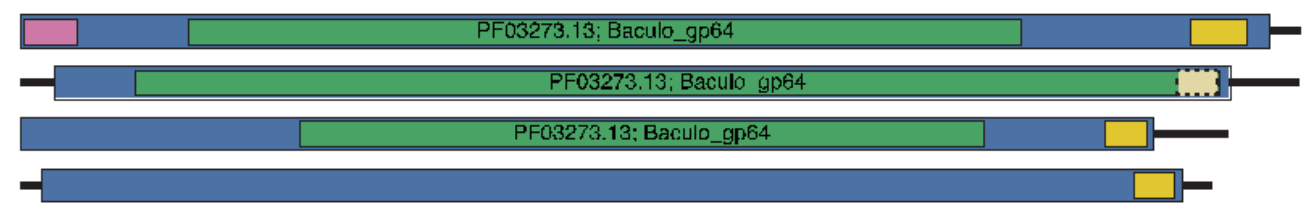

B) hypothetical1

11701 WMVo

1168 | Ūsinis

1160 | Astopletus

1181 I GMQLV1

C) hypothetical2

11701 WMVÉ

1168| Osinis

1160 | Astopletus

1181 I GMQLV1

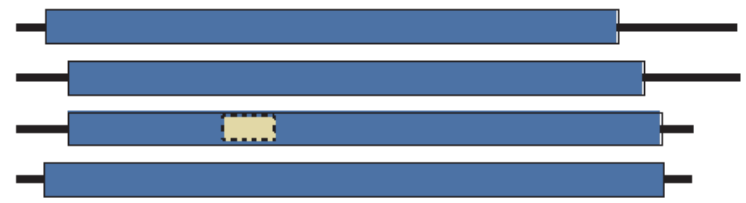

D) hypothetical3

1170 | WMV6

$1168 \mid \bar{U}_{\mathrm{sin}} \mathrm{s}$

1160 | Astopletus

1181 | GMQLV1

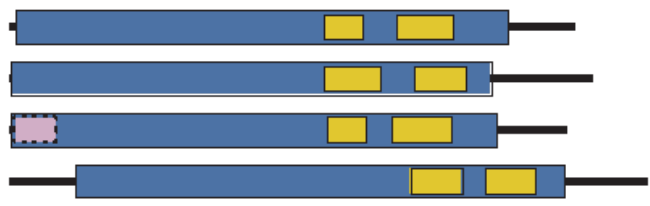

ORF

Pfam domain

transmembrane domain

signal peptide

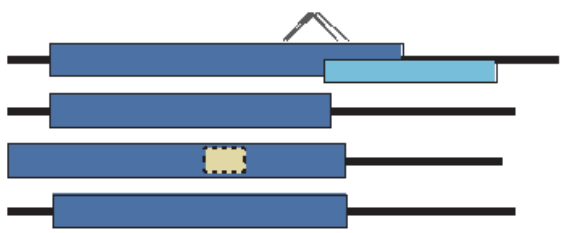

putative splice junction

E) Culex narnavirus 1

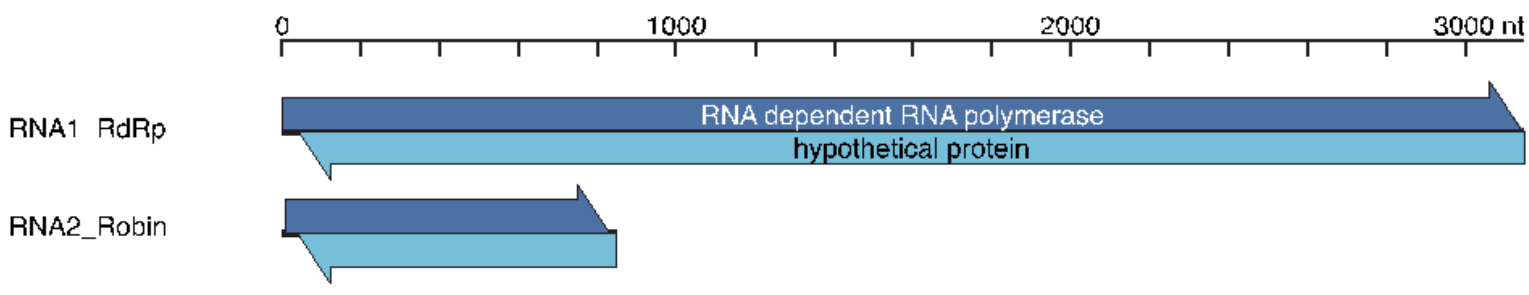

Supplemental Figure 8. Co-occurrence in individual mosquitoes allows identification of novel RNA genome segments in mosquito-borne viruses Diagrams of newly discovered genome segments for quaranjaviruses identified in this study, including Wuhan Mosquito Virus 6 (WMV6), Usinis, Astopletus, and Guadeloupe Mosquito, Quaranja-Like Virus 1 (GMQLV1) (panels A-D, respectively), and Culex narnavirus 1 (panel E), are shown (displayed 5' to 3', left to right, not aligned to each other). (A-D) The longest ORFs of the new quaranjavirus segments were analyzed for the presence of transmembrane domains and similarity to known domains (see Methods for details). Weaker support for these predictions is indicated by lighter color and dashed border. In the hypothetical 3 segment of WMV6, putative splicing events could result in a jump to a frameshifted ORF, which would substantially increase the coding region of the segment. (E) In both segments of the Culex narnavirus 1, long uninterrupted ORFs are found on both RNA strands (arrowheads indicate $5^{\prime}$ to $3^{\prime}$ direction), and in each case the codon boundaries are aligned. 
bioRxiv preprint doi: https://doi.org/10.1101/2020.02.10.942854; this version posted December 21, 2020. The copyright holder for this preprint (which was not certified by peer review) is the author/funder, who has granted bioRxiv a license to display the preprint in perpetuity. It is made available under aCC-BY 4.0 International license.
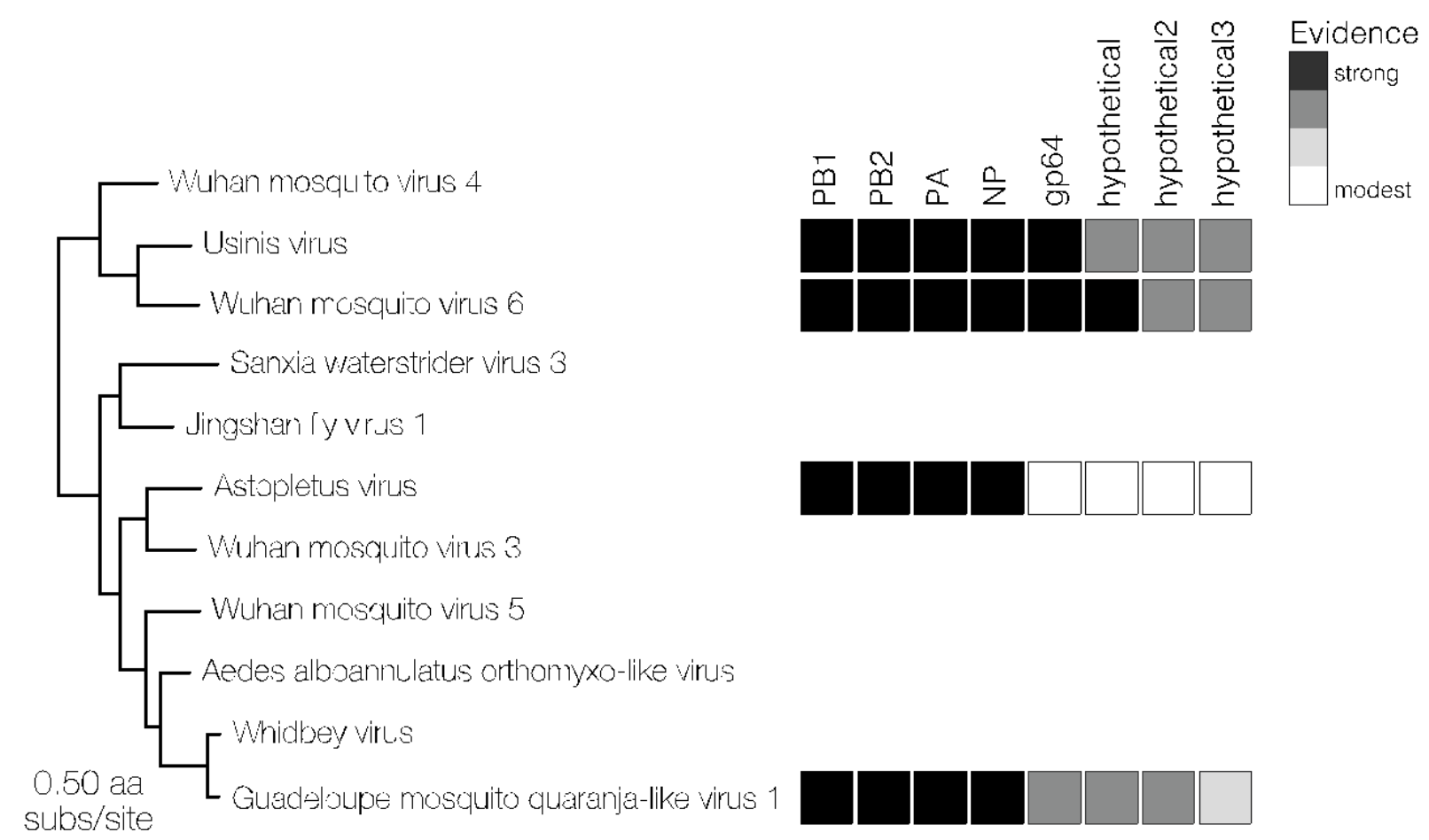

Supplemental Figure 9. RdRp-based maximum likelihood tree spanning the quaranjaviruses in this study for which 8 segments were recovered. The 8 boxes in the graphic to the right of the tree correspond to each of these 8 genome segments, which are labeled at top right. Levels of evidence for the existence of each segment is highlighted by a grayscale shading of the segment boxes, ranging from strong (black) to modest (white), based on: homology to BLAST hits from NCBI nt/nr database (black); assembly of contigs $>500$ nt from SRA samples containing the RdRp, or co-occurrence in $>10$ samples in our data (dark grey); reads mapping from SRA and co-occurrence in 5 samples in our data (light grey); or co-occurrence in $<5$ samples and shared protein domains (white). 
bioRxiv preprint doi: https://doi.org/10.1101/2020.02.10.942854; this version posted December 21, 2020. The copyright holder for this preprint (which was not certified by peer review) is the author/funder, who has granted bioRxiv a license to display the preprint in perpetuity. It is made available under aCC-BY 4.0 International license.
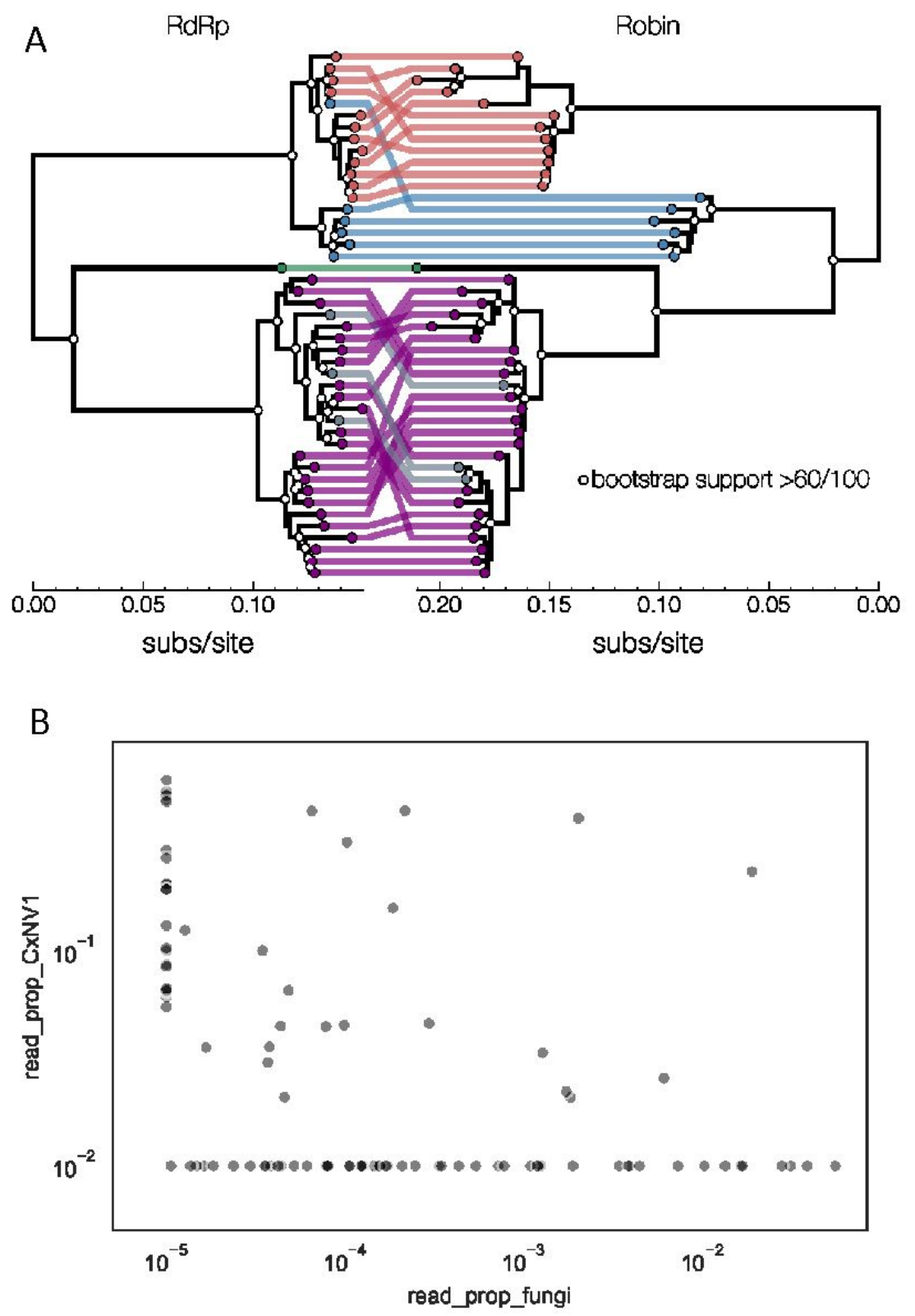

Supplemental Figure 10. Co-occurrence analysis enables identification of novel second narnavirus ambigrammatic RNA segment. (A) Narnavirus Robin segment. tanglegram shows two midpoint rooted nucleotide phylogenies of Culex narnavirus 1 RdRp segment on left and Robin segment on right. Tips are colored based on visual presence of clusters on longer branches clusters in red, blue, green, and purple are sequences reported in this study. Grey tips in the RdRp phylogeny were published by other studies, and their Robin segment counterpart was assembled here from corresponding SRA entries. Reassortment between the two segments is largely restricted to lineages within clusters, but reassortment also took place in either the red or the blue lineage. One possibility is a replacement of a red/blue lineage RdRp with a blue/red lineage or a replacement of red Robin with a diverged and unsampled lineage. White dots on nodes indicate bootstrap support $>60$ out of 100 replicates. Scale at the bottom of each tree is nucleotide substitutions per site. (B) Proportion of non-host reads mapped to contigs of Culex narnavirus 1 versus proportion of non-host reads mapped to contigs of any fungus. The lack of correlation argues against an indirect infection of fungi by the narnavirus. Each point represents a single mosquito. For visualization purposes, values of zero are displayed at $1 \mathrm{e}-6$ for fungi and $1 \mathrm{e}-3$ for Culex narnavirus. 
Table 1. Complete genomes of known and novel viral taxa recovered in this study

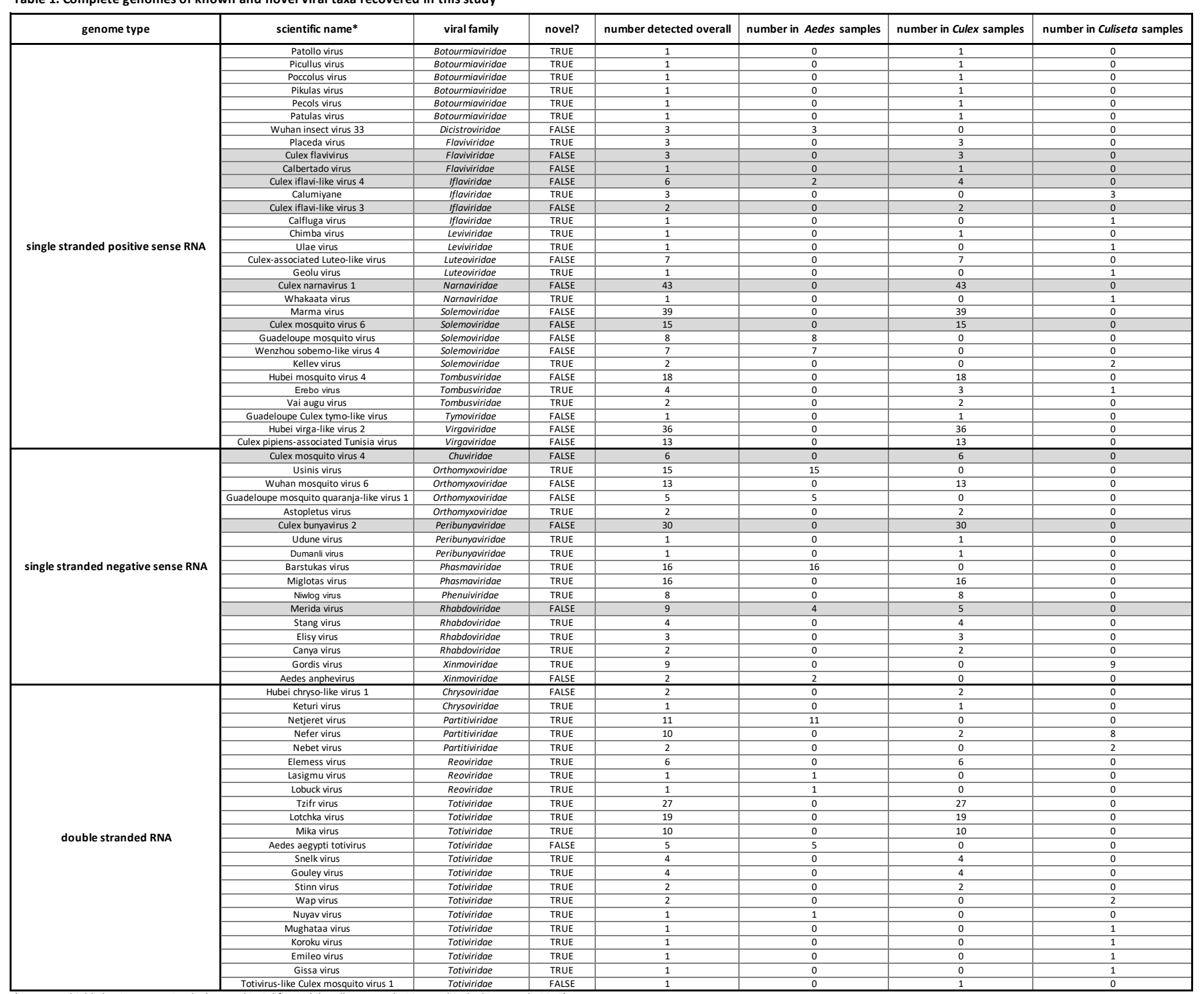

*gray rows highlight viruses previously detected in Califiornia (Chandler, JA et al., 2015 and Sadivghi, M et al., 2018) 